\title{
Use of Familiar Language to Enhance Reading Skills in Learners with Mild Intellectual Disabilities in Early Grades Primary Schools in Southern Province of Zambia
}

\author{
Kasebula Francis*, Beatrice Matafwali, Dennis Banda \\ The University of Zambia, Lusaka, Zambia
}

*Corresponding Author: Kasebula Francis, The University of Zambia, Lusaka, Zambia

\begin{abstract}
The Ministry of General Education introduced the Primary Language Policy in the 2013 curriculum framework that promotes the use of a familiar language in the lower grades (1-4) with a view to addressing the low reading levels among children in the lower grades as established by most researchers in the country. The implementation of the policy was based on the evidence from previous research which revealed that children learn literacy skills faster and easily, when taught in a language that is familiar to the child. However research revealed that the use of familiar language enhances the development of emergent literacy skills among children in the lower grades. However, there is no empirical evidence that the use of familiar language enhances literacy to learners with mild intellectual disabilities. Based on this premise, this study sought to investigate on the reading levels of children with intellectual disabilities with the goal of examining the extent to which the new policy addresses the reading skills of children with mild Intellectual Disabilities and to suggest effective teaching strategies. The study was a longitudinal study, conducted from 2018 September to 2019 September. Qualitative and quantitative research methods were used. Data collection was based on the questionnaire teachers teaching children with intellectual disabilities Basic Skills Assessment Tool (BASAT) was administered to learners with mild intellectual disabilities, structured interview schedule for parents and head teachers from the selected schools and observation of the learning environment in the selected schools. Quantitative data was analyzed by the use of IBM SPSS 25 version while qualitative data was analyzed by drawing themes from the data collected through the questionnaires and interview schedules. The results of the research revealed that there is no significant difference in the reading levels between the first data collected and the second respectively. The conclusion of the study was that the use of familiar language to teach literacy to learners with mild intellectual disabilities has not enhanced reading skills to learners with mild intellectual disabilities.. The researcher also recommends the implementation of the sound letter object identification
\end{abstract}

Keywords: familiar language, mild intellectual disability, reading

\section{INTRODUCTION}

This chapter provides background information, statement of the problem, purpose of the study, objectives, study questions, significance of the study, delimitation, and limitation of the study and the definitions of some terms used in the study. The methods employed in data collection and analysis of data which involved both qualitative and quantitative methods. Collecting data from respondents, questionnaires, interview schedules and focus group discussion were used respectively.

Reading efficiency plays an important role in the academic advancement of learners. Hence, it is very important that there should be deliberate policies and reading materials that can stimulate the development of emergent literacy skills in all learners, including those with mild intellectual disabilities. Mild intellectual disability is looked at in two skills, intellectual functioning (cognitive skills) and adaptive skills. The average intelligence quotient (IQ) is 100, therefore, the intelligence quotient (IQ) between 70 to 75 during diagnosis by professionals is considered to be intellectual disability. In view of the importance of reading and the low reading levels among learners, the Zambian government came up with the new approach to teaching reading using the familiar language to learners from grade one to four (1-4). This is in view that the knowledge and skills acquired from the familiar language will complement on the acquisition of reading skills in English language at higher levels of Education. However the empirical evidence to this effect is based on learners without 
intellectual disabilities. In the recent past, a lot of research has been conducted in order to validate the benefit of using familiar language to first graders as a way to enhance reading skills; it is against this background that this study sought to find out if learners with intellectual disability have benefited from the use of familiar language to learn reading. The policy on education recognises the use of familiar Zambian languages as the official languages of instruction in the Pre-Schools and early Grades (Grades 1-4). All the teaching and learning in all the learning areas at the Lower Primary level will be in familiar Zambian Languages. This is because there is evidence that children learn more easily and successfully through languages that they know and understand well. English will be offered as a subject, beginning at Grade 2. After the children have acquired sufficient literacy skills in the familiar language, it will be easier for learners to transfer these skills quickly and with ease to Literacy in English at Grade 2.

In view of this consideration, learners in Pre-Schools and lower primary (Grades $1-4$ ) will be given an opportunity to learn not only the initial basic skills of literacy and numeracy in a familiar language but also all knowledge, skills and values in the other learning areas. In Zambia, the seven (7) zone languages; Cinyanja, Chitonga, Icibemba, Kiikaonde, Lunda, Luvale and Silozi as well as the widely used community languages in specific school catchment areas will be used for this purpose. It should also be noted that the use of a familiar language should be extended to learners with Special Educational Needs. English will still remain as the official medium of instruction beginning at Grade 5 up to tertiary.

It is hoped that this approach will foster better initial learning, enhance the status of Zambian Languages and integrate the schools more meaningfully into the life of the local communities (Curriculum frame work, 2013). Reading is one aspect in the learning process that plays a significant role in the academic success of every learner and hence the need to lay a strong literacy foundation in the learners at an early stage of their education

\section{BACKGROUND TO THE STUDY}

The colonial and Federal governments favored the use of familiar language to teach in the early years of school. Dutton (1965) defines language as a system of arbitrary and vocal symbols by means of which a social group cooperates with one another. After independence, the medium of instruction changed to English from grade one to the highest level of education which is the University (Linchen, 2004).This implies that the people who introduced western education in Zambia had a clear reason why they thought of using the local language to teach literacy skills at lower grades as effective than using the second or foreign language to teach reading skills to children at an early stage of learning. This is the same concept which the Ministry of Education is implementing in an effort to enhance reading levels among early graders in schools.

The low literacy levels among Zambian school going children has been a major policy concern in the last decades. The study conducted by the Southern Africa Consortium for Monitoring Education Quality (SACMEQ) II in all the nine provinces of Zambia, revealed that $25 \%$ of grade six pupils could not read at a minimum level of proficiency and only $3 \%$ could read at a specified desirable level ((MOE, 1995).The findings of the Zambia National Assessment Survey (ZNAs) (2016), is in consistent with the findings of the SAQMEC and EGRA on the low reading levels of most learners at the lower grades and that this includes children with mild intellectual disabilities. Further still, the research reports by William, (1993), Mwanza,(2012), Kasebula (2013), Matafwali, (2010) and Lwangala, (2011), also revealed that on average pupils could not read text two levels below their own grade level. The reason to this was attributed to the use of unfamiliar language (English) when teaching literacy. Kelly (1995) adds that most backwardness in reading among learners at lower levels is as a result of the language factor. The studies by SACMEQ involved learners without intellectual disabilities from grade five (5) to six (6) however, the situation of low reading abilities could be worse off among learners with intellectual disabilities. A compendium of examples in the SACMEQ 111, (2011) report, King and Mackey, (2007) and UNESCO (2018), has attested to the low reading levels among children in early grades. However, the benefits of using familiar language as medium of instruction to teach literacy skills to early graders is based on the premise that children learn more easily and successfully through languages that they know and understand well (Curriculum frame work, 2013).The Educating Our Future policy document (1996), Tambulukan and Bus, (2011), 
MOESVTEE,(2013) also emphasize on the use of a familiar language predominantly spoken in an area to teach literacy as a way to enhance literacy levels among learners with intellectual disabilities and without intellectual disabilities. Benefits of language and culture-rich curriculum for Native children: Academic success: Based on years of research, the inclusion of Native language and culture in school curriculum is an important factor in Native children's academic achievement, retention rates, and school attendance, (Demmert, 2000; Lipka and McCarty 1994; McCarty and Lee, 2014; Mmari, Blum and Teufel, Shone, 2010; Skinner 1999; Yazzie, 1999). Gay (2013) notes that a culturally responsive approach to teaching reading connects students' experiences in and out of the school, supports educational equity and excellence, and empowers learners by giving them the skills to effectively negotiate and impact the world around them. This type of teaching approach and the inclusion of indigenous languages are not detrimental to academic achievement; rather, it promotes academic achievement and cultural knowledge, preparing youths to be leaders of their communities (McCarty \& Lee, 2014).

Research conducted in the recent past has revealed that use of familiar language instructions improves overall school performance, cognitive development, problem solving, and creativity. The National Adult Literacy Survey (NALS), conducted in 2004, in the United States of America, showed that low literacy is a common problem in the United States. In Britain, a study conducted by the Organization for Economic Cooperation and Development (OECD) in 2001, indicated that about seven million adults are unable to read, write or add up to the level they would need to communicate in the world of business (Garner, 2001\&OECD, 2001). Waikeman and Rickemalman (2006) also reports that most recent research tends to focus much on reading instructions rather than the prevalence's of reading among children with intellectual disabilities. Katims (2001) examined 132 students with intellectual disabilities in Texas and found $22 \%$ of them showing minimal abilities to read. In Zambia, the National Assessment Survey conducted in 2006 indicated that the national mean performance in reading in English among grades 5 pupils was 34.5 percent compared to 33.9 percent in 2003 survey and 37.30 percent respectively in (2016). The findings in the survey resonates consistently with what Matafwali (2010) study revealed that despite the existence of a rich literacy program in Zambia, the reading levels of the majority of Zambian children was remarkably low.

Kelly (1995) adds that most backwardness in reading is as a result of the language factor. Benefits of using the familiar language as medium of instruction to teach literacy skills to early graders is based on the premise that children learn more easily and successfully through languages that they know and understand well (Curriculum frame work, 2013).The Educating our Future policy document (1996) also emphasize on the use of a familiar language predominantly spoken in an area to teach literacy as a way to enhance literacy levels among early graders. The significance of reading in the academic advancement of every pupil cannot be over looked if pupils are to progress in their education career. Banda (2008) also argues that the attainment or achievement of quality and meaningful education cannot be attained only through formal education and suggests that the hybridization of African indigenous knowledge systems (AIKS) can enhance learning. Government of the republic of Zambia through the Ministry of Education has come up with the education policy on the teaching of reading to early graders using familiar language with the view to enhance literacy skills to learners. It is hoped that learners can build up on the skills earlier on acquired in familiar language even as they start learning in English as a second language. The enhancement of reading is very important because reading play a critical role to the academic advancement and social well-being of any individual regardless of disability or gender (Matafwali, 2005). Kashoki, (2019) further states that the general picture that is evident is one that shows that while a part of teaching process is devoted to enabling the learner to gain a practical speaking, reading and writing knowledge of the language is critical in every sphere of life of an individual.

\subsection{Global Perspective}

Research conducted in the recent past has revealed that familiar language instructions improves overall school performance, cognitive development, problem solving, and creativity. Bilingual children have increased met linguistic awareness, or knowledge 'about' languages. These Meta linguistic skills are an important piece of intellectual development, reading skills development, and overall academic success (Hakuta, 1986). Meta linguistic abilities are seen in greater phonemic awareness in bilingual children (for example, they can answer questions like "Do cat and car have the 
same sound at the beginning?"), and in bilingual children's ability to break words into syllables more successfully than monolingual children (Lindfors, 1991). The use of familiar language and culturerich curriculum for Native children does not only benefit children without disabilities but also learners with intellectual disabilities, (Blum and TeufelShone, 2010; Skinner 1999; Yazzie, 1999). Gay (2013) notes that a culturally responsive approach to teaching reading connects students' experiences in and out of the school, supports educational equity and excellence, and empowers learners by giving them the skills to effectively negotiate and impact the world around them. This teaching approach and the inclusion of indigenous languages are not detrimental to academic achievement; rather, it promotes academic achievement and cultural knowledge, preparing all learners to be leaders of their communities (McCarty and Lee 2014).

The problem of low reading levels is not unique to developing countries such as most African, Asian and South American countries. The National Adult Literacy Survey (NALS), conducted in 2004, in the United States of America, showed that low literacy is a common problem in the United States. In Britain, a study conducted by the Organization for Economic Cooperation and Development (OECD) in 2001, indicated that about seven million adults are unable to read, write or add up to the level they would need to communicate in the world of business (Garner, 2001:OECD,2001). Current research is demonstrating the effectiveness of comprehensive reading instruction for individuals with intellectual disabilities Allor, Mathes, Roberts, Cheatham, and Champlin, 2010; Allor, Mathes, Roberts, Jones, and Champlin, 2010; Browder, Ahlgrim-Delzell, Courtade, Gibbs, and Flowers, 2008). One overarching finding from these studies is that intense amounts of repetition and practice on critical literacy skills are needed to produce meaningful gains among learners with intellectual disabilities. Providing intensive instruction in the school setting is extremely challenging. The other ultimate aim of this study will be to explore the various strategies that can be used to intensify early literacy instruction for students with intellectual disability, including methods for providing practice outside of teacher-led instruction. The most fundamental job of this nation's education system is to teach children to acquire competencies and skills in literacy and numeracy as outlined in the curriculum frame work, (Curriculum Frame Work, 2013). In spite of this manifesto, teaching children with intellectual disabilities (ID) to read has been largely ignored in the national rhetoric. Typically it has been assumed that reading is a skill beyond the intellectual capabilities of most students with intellectual disability (ID) and that at best they might be taught to recognize a limited number of sight words (i.e., high frequency words). As such, four in five children with mild to moderate intellectual disability never achieve even minimal levels of literacy (Katims, 2001). Recent research supports the view that intellectually disabled students should be taught to read in a manner similar to other students who struggle to learn to read (Allor, Mathes, Roberts, Cheatham, et al., 2010; Allor, Mathes, Roberts, Jones, et al., 2010; Browder et al., 2008). Specifically, students with intellectual disability should be taught to read using strategies and techniques that will provide them with the skills necessary to fully process individual words in connected text and derive meaning from the text. These findings above suggest that when given scientifically based and rigorously intensive reading instruction in familiar language, over an extended period of time, these children respond positively, making important gains in literacy development. Instructions must be systematic and explicit, including all reading components; repetitive in its use of routines and instructional language; fast paced; and highly motivating. Lessons should be fully implemented by teachers skilled in effective reading instruction. Learners with intellectual disability require large amounts of repetition to make meaningful progress. Increasing practice of critical skills is extremely challenging. Resources are finite; therefore, educators must find feasible methods for increasing the intensity of interventions. The purpose of this study was to investigate the effectiveness of using familiar language to teach reading to learners with intellectual disabilities. Research reveals that children learn very well when teachers use the familiar language of the children to teach reading and that parents or guardians are likely to take an active role in the education of their intellectually disabled children. Intensive instruction is required to provide all children with the opportunity to develop the skills necessary to become literate, these studies are a clear reflection of the low reading levels among pupils with and without disabilities. Wagner and Torgesen (1987:209) suggested that "phonological awareness may be difficult to train' moreover the notion of developmental appropriateness could complicate teaching young children with intellectual disabilities. Due to different levels of intellectual disability, teaching reading to pupils could be a challenge to most teachers'. However as revealed by the Green paper of (1997), Excellence for All 
Use of Familiar Language to Enhance Reading Skills in Learners with Mild Intellectual Disabilities in Early Grades Primary Schools in Southern Province of Zambia

Children, that capacity building of teachers and schools to teach reading is vital to raising the achievement of learners with intellectual disabilities. Research has shown that children's first language is the optimal language for literacy and learning throughout primary school (UNESCO, 2008a).

\subsection{Zambian Perspective}

In Zambia, the National Assessment Survey conducted in 2006 indicated that the national mean performance in reading in English among grades 5 pupils was 34.5 percent compared to 33.9 percent in 2003 survey. The study revealed that pupils were able to deal with material presented successfully (Zambia's National Assessment Survey Report, 2006). The study by Matafwali (2010) revealed that despite the existence of a rich literacy program in Zambia, the reading levels of the majority of Zambian children was remarkably low. This study further revealed that 50 percent of grade one and two pupils involved in the study scored zero or only one word on the reading subtest. This phenomena show that the literacy levels among school going children is very low from the expected standard and it could be worse to learners with intellectual disability. This is the reason why this study aimed at investigating on the reading levels of learners with intellectual disability after implementing the teaching in a familiar language, for a period of two academic years.

MOE (1996) policy document points out strongly on the importance of reading and writing to pupils in the lower and middle grades. The policy document states that "the fundamental aim of the curriculum for the lower and middle basic classes 1 to 7 is to enable pupils to read and write correctly and correctly in the Zambian language and English". (MOE, 1996:34). This is also in line with what Kashoki, (2019) states as he urges governments and higher learning institutions to promote the use of African languages as a tool to enhance economic and social development. This also explains the stance that Ministry of Education has taken to improve the reading levels of all pupils in the country. The new curriculum frame work (2013) has explained on how the ministry wants to enhance reading to early graders by using familiar language to teach children in the lower grades, learners with intellectual disabilities are not an exception. This assertion is advocated for on the premise that children learn well in the language they are familiar to. The curriculum framework (2000:21) states that the language of instruction in school is a pedagogical too for explaining, communicating asking and responding understanding and activating the learning process. This explains the significance of use of familiar language to teach reading skills to children with intellectual disability in the lower grades. However benefits of this approach to learners with intellectual disability are not known as most research is conducted on learners without intellectual disabilities at the expense of the intellectually disabled learners. Therefore, it is for this reason that this study investigated the effectiveness of using familiar language to teach reading skills to intellectually disabled learners and ascertain the extent to which literacy levels have been enhanced among learners with intellectual disabilities.

\subsection{Statement of the Problem}

Reading levels among early grade learners are substantially low as revealed by the studies conducted by the Ministry of Education, 2011, Matafwali (2005,2010), Kasebula, (2013),National Assessment Survey of Learning Achievement in Grade 2 in Zambia, (2015,2016); Nkamba, and Kanyika, (1998). A research by Luangala (2011) reported that learners were reading at three grade levels below their own level. Therefore, the Ministry of Education has introduced the use of familiar language to teach early literacy in the early grades, based on the fact that children, including those with mild intellectual disabilities learn well when familiar language is used as a mode of instruction. Research has revealed that most children's reading levels have improved since then, Tambulukani and Bus (2015). Research by Christopher Ratz in (2013) in Germany revealed that $29.35 \%$ of children with mild intellectual disability do not read at all while $6.8 \%$ read at logographic. Whilst the reading levels remain low, there is no empirical evidence to show that the use of familiar language to teach children with mild intellectual disabilities has improved their reading ability or not. Hence it is against this background that this study sought to investigate whether the new approach to teaching reading in a familiar language to learners with mild intellectual disabilities has enhanced reading or not and to recommend teaching strategies of reading to learners with mild intellectual disability.

\subsection{Purpose of the Study}

The purpose of the study was to investigate the benefits of using familiar language to teach reading to learners with mild intellectual disabilities. Further, the study has also recommended effective teaching strategies to enhance reading among the learners with mild intellectual disabilities. 
Use of Familiar Language to Enhance Reading Skills in Learners with Mild Intellectual Disabilities in Early Grades Primary Schools in Southern Province of Zambia

\section{OBJECTIVES OF THE STUDY}

\subsection{General Objective}

The general objective of this study was to investigate on how the new language policy of teaching using familiar language to enhance literacy levels among early graders has enhanced reading skills to learners with intellectual disabilities.

\subsection{Specific Objectives of the Study}

- To establish whether the use of familiar language to teach reading to learners with intellectual disabilities is credible

- To determine the reading skills that could have been enhanced due to the use of familiar language

- To asses if the new approach to teaching reading has enhanced parental involvement in the learning of learners with intellectual disability.

- To establish the challenges of the language policy on learners with intellectual disabilities.

- To investigate on the most effective teaching strategy of teaching reading to learners with intellectual disabilities.

\subsection{Study Research Question}

How beneficial has the use of familiar language to teach reading been to learners with intellectual disabilities?

\subsubsection{Specific Questions}

- How credible has been the use of familiar language to enhance reading to learners with intellectual disabilities?

- Which reading skills have been enhanced as a result of using familiar language to teach reading to learners with intellectual disabilities?

- Has the use of familiar language enhanced parental involvement in the learning of learners with intellectual disabilities?

- What challenges have you observed in teaching reading to learners with intellectual disabilities as a result of using familiar language?

- Despite the challenges observed in the use of familiar language to teach reading, what could be the effective teaching strategy?

\subsection{Significance of the Study}

The significance of this study was to investigate and establish and bring to light authentic information on the improvements that the new approach to teach literacy has made to enhanced reading among learners with intellectual disabilities and also to suggest effective teaching strategies of reading. Most research findings in the recent past such as SACMEQ 111, (2011) report, King and Mackey, (2007) and UNESCO (2018), point out to the fact that the use of familiar language to teach literacy has brought about significant improvements to the reading levels among learners. However this assumption is based on learners without intellectual disabilities and not specifically learners with intellectual disabilities. The findings of this research might help stake holders such as the Ministry of Higher Education to consider implementing certain mitigation measures to teach literacy to learners with intellectual disabilities.

\subsection{Delimitation of the Study}

The study area for this study was as follows; Sinazongwe district (Maamba Special School), Choma district (St Mulumba Special School), Livingstone district (Mujala Special Unit, Nakatindi inclusive school and David Livingstone Secondary Special Unit). The study will be confined to the stated schools only for the reason being that it is where the required population is found in the southern region of Zambia. 
Use of Familiar Language to Enhance Reading Skills in Learners with Mild Intellectual Disabilities in Early Grades Primary Schools in Southern Province of Zambia

\section{CONCEPTUAl Frame WORK}

A coherent idea presented in a systematic form constitutes a concept. Huberman and Miles (1994), define a conceptual frame work as a written or visual presentation that explains phenomena in a narrative form or diagrammatic form. This study is anchored on the concept that children with intellectual disabilities learn concepts easily and faster if concepts are presented in a familiar language, meaning that much of the learning should be reality based. This will attach a lot of meaning to what they learn and in this way they are likely to acquire the reading skills when taught in a familiar language consistently. Below is the diagrammatic representation of the key factors of the concept of how the social cultural interactions influence has on the emergent literacy skills of children with intellectual disabilities, (Paula, 1998).

\section{CONCEPTUAL FRAME WORK}

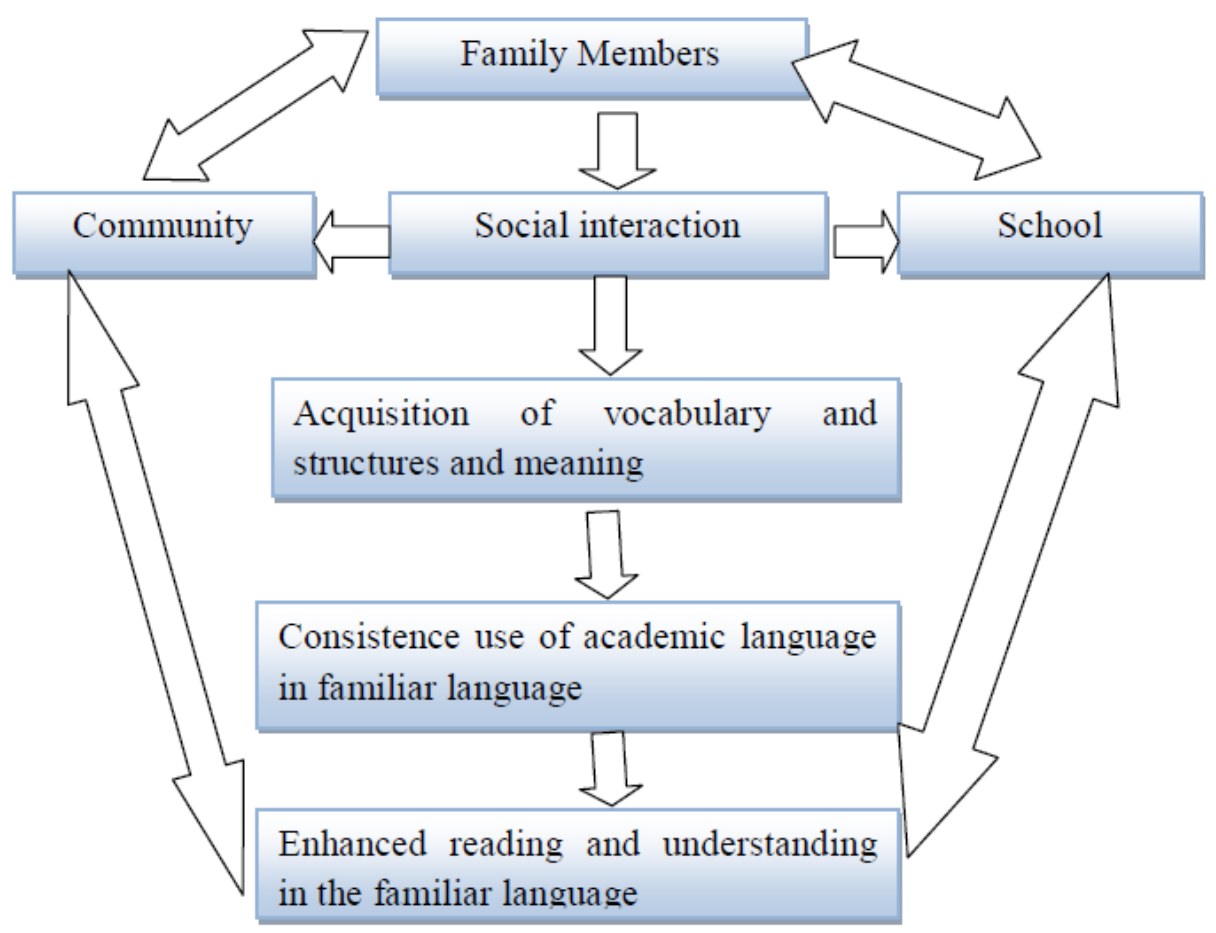

Source: Kasebula (2019)

The above representation of the framework shows that there are a multiple linkages in the social cultural interactions in the community, homes and school and that in between these interactions, there is a rich interchange of knowledge among children and adults. It is in this interface of knowledge exchange that learners with intellectual disabilities stand to benefit in the acquisition of rich vocabulary in a familiar language, which is vital for their learning at school. The frame work identifies peers in the community, parents/relatives and siblings, community members and the teachers at school, as key stake holders in the development of reading skills in learners with intellectual disabilities. The assumption for parental involvement in the learning of their children is that, they play a very important role to bridge the gap between school and community. Catherine et al, (2005), states that "an encouraging home literacy environment can produce better results in the acquisition of reading skills in learners.

The conceptual frame work followed in this research aims at strengthening the focus of teaching of reading to children with mild intellectual disabilities by use of familiar language, which is reality based approach. On the other hand, the framework acknowledges the Sound Letter Object Identification (SLOI) as being an effective teaching approach as opposed to the traditional Letter Sound Knowledge (LSK) approach, which encourages children to memories the alphabet song. My argument is that using the (SLOI) approach to teaching reading in a familiar language to children with intellectual disabilities, children will be able to identify the objects being referred to and familiarize with the name. This can even be the other way round, where children can identify a word and relate it to the object because it is familiar to them. The epistemological assumption to this effect 
is that the research findings will influence the outcome, which is objective about phenomena. This assumption will also bring about the justification of using both quantitative and qualitative research methodologies.

\section{THEORETICAL FRAMEWORK}

The Early Literacy Development (ELD) framework embraces Vygotsky's theory that cognitive and linguistic development is socially constructed and that children's development can be "led" through appropriately mediated instruction (Berk and Winsler, 1995; Bodrova and Leong, 2007; Copple and Bredekamp, 2009). Vygotskian theory emphasizes that children's development is best understood within the cultural, social, and historical contexts in which it occurs ( Berk, Winsler, 1995 and Garcia, Markos, 2013). Vygotsky social cultural theory of human learning describes learning as a social process and the origination of human intelligence in society or culture. The social cultural theory of human learning describes learning as a social process and the origination of human intelligence in society and culture. The major theme of the theoretical frame work is that social interaction plays an important role in the development of cognition. Therefore the use of familiar language to teach reading provides effective scaffolding by teachers, parents, care givers and peers to support the learning of children with intellectual disabilities. The social interplay through the use of familiar language creates a better environment for cognitive development, leading to the development of most cognitive skills such as reading for academic advancement. This epistemological argument explains the reality or justifies the belief that learners learn well and faster when they learn in a language they are familiar to.

\subsection{Definition of Terms}

- Intellectual Disability: Sub average intellectual functioning of an individual concurrent with deficits in adaptive behavior and manifested during the developmental period.

- Reading: The process of identifying and understanding the meaning of the characters and words in written or printed materials

- Phonological Awareness: This is the ability to recognize that a spoken word consists of smaller units.

- Effectiveness: The significant role that familiar language has on enhancing reading skills to learners with intellectual disabilities.

- Familiar Language: The language that a learner recognizes and understands easily and uses it on a regular basis.

- Mild Intellectual Disability: Intelligence quotient (IQ) of a child between 60-75

\subsection{Summary}

Chapter one has highlighted the global and local perspective on the reading levels of learners. From the previous studies, it is quite clear that the reading levels of most learners are remarkably low and hence the need for this study so as to establish the positive benefits that learners with intellectual disabilities accrued due to the use of familiar language by teachers to teach reading to learners with intellectual disabilities. This chapter has also highlighted on the social cultural theory by Vygotsky as a guide to the study.

\subsection{Literature Review}

This chapter is going to reveal different literature in line with the themes generated from the study objectives. Both local and international literature shall be consulted for in-depth understanding of the topic under study. The aim is to establish the information gap which this study seeks to fill in the education fore.

\subsection{Effectiveness of Using Familiar Language to Teach Literacy}

The Introduction of Language of instruction is one of the primary determinants of effective classroom communication. Globally, many countries with multiple languages have required a single language to dominate their education sectors (UNESCO, 1953, 2003). This phenomenon is particularly prevalent 
in Africa. Depending on different estimates and definitions, the number of languages spoken in Africa ranges between 1,000 and 2,500 (Gadelii, 2004). However, only 176 of these African languages are used in education, and for many languages, their use is often limited to informal education programs (UNESCO, 2010). In formal education, most African countries use the language of their historical colonizers or the language of a dominant ethnic group. The use of unfamiliar languages as a medium of instruction is often mentioned as an important source of low enrolment rates, retention rates, literacy levels and academic achievement (Tihtina, Zenebe Gebre, 2014).It is against this understanding that government of the republic of Zambia thought of introducing the use of familiar or local language in order to enhance literacy levels among the early graders. Zambia is widely claimed to have over 72 languages, although many of these might be better regarded as sublanguages, because they originate from other major languages. All of Zambia's vernacular languages are members of the Bantu family and are closely related to one another. This is supported by Sepell, (2014), where he states that in Zambia the indigenous languages all belong to the Bantu taxonomic groups within which varieties share strong core of grammatical and lexical commonalities and the boarders within them are porous. Only 7 languages are used as lingua franca in the ten regions of Zambia and are used for learning purposes". Using familiar language to teach in early grades is important as also observed by the Global Education and Monitoring Report of 2016. Further still Sepell and Simatende (2016) contends that the A,E,I,O and U, each consonant represents a single phoneme in each of the Bantu languages of Zambia and this makes initial literacy learning easier than it is in English where each of the letters represent different phonemes. The 7 languages official languages in Zambia together represent the major languages of each province: Bemba (Northern Province, Luapula, Muchinga and the Copperbelt), Nyanja (Eastern Province and Lusaka), Lozi (Western Province), Tonga (Southern Province), and Kaonde, Luvale and Lunda (Northwestern Province), (Ayo Bamgbose, 1991). However, from 2000 census report, Zambia's most widely spoken languages are Bemba (spoken by $52 \%$ of the population as either a first or second language), Nyanja (37\%), Tonga (15\%) and Lozi $(11 \%)(\mathrm{CSO}, 2000)$. A retrospective look at the use of African languages as languages of instruction in schools will show that much progress has been made over the years. Research on Quality Education In (1996), government of Malawi announced an extension of the existing school language policy. In a letter that was circulated to all Regional Education Officers, District Education Officers and heads of other educational institutions, government stated that, with immediate effect all standards 1,2, 3 and 4 classes in the schools be taught in their own mother tongue or vernacular language as a medium of instruction. According to a follow-up government press release on this new school language policy, vernacular language and mother tongue was defined as - language commonly spoken in the area where the school is located (Ministry of Education: 1996). The reason that was given in this press release for making this extension to the school language policy was twofold. It was stated that this new language policy would give children an opportunity to participate fully in classroom discussions using a language that they are more familiar with than to struggle in a language that they are just learning (Henri Chilora, 2000). A separate study by the Zambian Ministry of Education under the auspices of the Southern African Consortium for Monitoring Educational Quality (SACMEQ) in 1995 (report published in October 1997) showed that only 25\% of Grade 6 pupils could read at defined minimum levels and only $3 \%$ of grade 5 pupils could read at defined desirable levels. It was evident that the first thirty years of English medium had been less than satisfactory. Teaching and learning in an alien language had meant that, for the vast majority, school was unrelated to real life. Rote learning was the only way to approach a situation where understanding was absent from school, with mindless repetition replacing problem solving and inventiveness. By 1995, there was a growing awareness within the Ministry of Education that reading and writing were better developed first in a language with which children were familiar. It follows the basic principle of working from the known to the unknown, i.e. learning first in a known language (L1) and later moving into the unknown (L2), enables pupils to express themselves in a meaningful way and therefore participate in their own learning processes, it also prevents cognitive overload in pupils, since they are concerned with only one thing at a time, that of learning to read and write in a familiar language instead of having to negotiate both the reading skill and the new language, it reinforces pupils ${ }^{6}$ self-esteem by validating their cultural identity. It is also hoped that learners with intellectual disabilities can also benefit by learning in a familiar language. The ontological and methodological epistemological assumption to the use of familiar language to teach literacy to learners with intellectual disabilities is also strengthened by Vygotskys cultural theory of learning. Children, who learn literacy skills in local 
language and then English (Bilingual), have increased meta-linguistic awareness, or knowledge 'about' languages. These meta- linguistic skills are an important piece of intellectual development, reading skills development, and overall academic success (Hakuta, 1986). Meta-linguistic abilities are seen in greater phonemic awareness in bilingual children (for example, they can answer questions like "Do cat and car have the same sound at the beginning?"), and in bilingual children's ability to break words into syllables more successfully than monolingual children (Lindfors, 1991). This implies that there is enough evidence that use of familiar language in the education of learners with and without disabilities, have more benefits in academic advancement and better understanding of concepts at higher grade levels, however little is mentioned on the effectiveness of the approach to learners with intellectual disabilities. A child with a language difficulty or learning disability can learn a second language, and becoming bilingual is not a hardship on a child with disabilities, including languagespecific disabilities. However, a child with a learning disability will still have that disability when learning more than one language; a bilingual child with specific language disabilities (SLD) will still be slow acquiring both languages, (Edwards, 1989). Acquiring concepts and skills in English will not be difficult for learners with intellectual disability though very little or nothing at all is mentioned about the enhancement of reading skills other than the acquisition of the second language without much difficulties. The social learning theory by Bandura, (1977) suggests that humans can learn automatically through observation rather than through personal experience. This suggests that learning can be enhanced through the use of familiar language as it adds meaning to what is head and observed.

Lack of reading skill limits one's quality of life (Bradford, Shippen, Alberto, Houschins, and Flores, 2006) and yet only 1 in 5 students with intellectual disabilities reach minimal literacy levels (Katims, 2001). Slow development of reading skills may affect more than just one academic subject but may also delay language acquisition, general knowledge, vocabulary, and even social acceptance. The development of literacy skills in elementary school can also have long-term effects. Thus, successful early readers usually succeed academically while those who fail to learn to read in elementary school frequently have lifelong difficulties in learning new information (Matthew 4 effect: education, 2013). Educators have called this the Matthew effect, a term based on the Biblical message found in Matthew 25:29 and paraphrased as "the rich get richer and the poor get poorer" (Matthew effect: education, 2013). While assistive technology may become more prevalent in everyone's world, reading will continue to be an essential part of school, work, and community life. Educators working with students with intellectual disabilities have many reasons to include literacy instruction in their classrooms. Reading and literacy skills are the key to accessing knowledge, gaining independence, and making choices (Houston and Torgeson, 2004). Also, the Individuals with Disabilities Education Act (IDEA) of 1997 (PL 105), most recently amended in 2004, is a federal law requiring all students to have access to the general education curriculum. Since reading is part of that curriculum it should also be part of the curriculum for students with mild to moderate intellectual disabilities (Houston and Torgeson, 2004). Additionally, the No Child Left behind Act (NCLB) of 2001 (PL 107-110) requires that all students, including those with disabilities, achieve adequate yearly progress of grade level standards.

\subsection{Reading Performance by Children who Speak The Familiar Language used to Teach}

Speaking the language of instruction English is the language of instruction in most SACMEQ countries, except in Tanzania and Zanzibar, where Kiswahili is used, and Mozambique, where Portuguese is used. A few schools in South Africa use Afrikaans. It is of interest to note that past analyses of SACMEQ II data showed strong positive correlations between speaking the language of instruction and pupil achievement, especially in reading, across all SACMEQ countries. A comparative study was done by William on reading levels between lower grades in Zambia and Malawi. The study revealed that Malawians did better because the initial language of instruction was done in a familiar language, (William, 1993).

SACMEQ has conducted four major education policy research projects between 1996 and 2013. The recent data published by SACMEQ suggest South Africa (and a number of other countries) made tremendous improvements in the quality of its primary education between 2007 and 2013. Though results and its analysis is questionable, the improvements arise from different methodologies employed in 2007 and 2013, making them incomparable until they are properly equated," wrote 
education researcher and fellow at the Organization for Economic Co-operation and Development( OECD). The previous research project, conducted between 2006 and 2011, assessed 61,396 Grade 6 pupils and 8,026 Grade 6 teachers in 2,779 schools across 14 countries. The research reports in mention, where conducted on learners without intellectual disabilities and therefore it remains an assumption that children with intellectual disabilities learn well by the use of familiar language.

In Mali, as in other former French colonies in Africa, the challenges faced in education and in the development of African languages can be largely attributed to the legacy of French colonial policy. The historical dominance of the French language in the formal education systems despite the lack of French fluency among most of the African population has led to poor learning outcomes, teaching strategies that do not promote comprehension and the stunting of students' natural learning abilities. In 1979, the government of Mali took the radical decision to introduce the use of national languages in formal education as an attempt to solve critical problems of student abandonment, failure and grade repetition. Bilingual education began on an experimental basis; national languages were introduced in Grades 1 through 6 as the medium of instruction, in this government policy, there is no specific mention of teaching learners with intellectual disabilities by the use of familiar language as a way to enhance reading. The use of familiar languages as medium of instruction does not suffice to guarantee optimum effectiveness of teaching and learning. The use of national languages in basic education was first introduced in 1979, but nothing else in the curriculum, such as teaching methods and content of instruction, was changed. As a result, learning outcomes did improve for students in the experimental schools, but not a great deal. It was concluded that the use of the national languages in education could not be maximally successful without revising teaching methods and developing adequate teaching and learning materials. The transition from the national language to French process also needed specific attention. Thus, the use of national languages has been just one component of Pedagogic convergent. For optimum results to be attained in the use of familiar language to teach literacy therefore requires much more attention on the part of teaching methodology and production of learning materials for both learners with and without intellectual disabilities. Teaching and learning materials keep the teacher focussed on the curriculum and motivates learners to engage in the learning process. As a result, learners are likely to grasp and master the concepts. However positive results are evident in most research findings on the effectiveness of using familiar language to teach literacy skills to both learners with intellectual disabilities and the "normal".

The Program for the International Assessment of Adult Competencies (PIAAC) is a cyclical, largescale study that was developed under the auspices of the Organization for Economic Cooperation and Development (OECD). Adults were surveyed in 24 participating countries in 2012 and 9 additional countries in 2014.The goal of PIAAC was to assess and compare the basic skills and the broad range of competencies of adult literacy around the world. The assessment focused on cognitive and workplace skills needed for successful participation in 21st-century society and the global economy. Specifically, PIAAC measured relationships between individuals' educational background, workplace experiences and skills, occupational attainment, use of information and communications technology, and cognitive skills in the areas of literacy, numeracy, and problem solving. The survey revealed that most adults around the globe where lacking literacy skills to match with the demands of the $21^{\text {st }}$ century.This revelation has a trickledown effect on the children. If most parents lack the literacy skills in reading, how can they be so committed to help their children to learn to read and learners with intellectual disabilities are the most victims of low literacy levels world over.

\subsubsection{Zambian National Literacy Policy}

The purpose of the Zambian Education Literacy Guide is to establish a set of guidelines for teaching and learning literacy in all Zambian schools. Zambia has had experience with traditional teacher directed methodologies as well as the more participatory Primary Reading Program (PRP). The curriculum has moved away from the Whole Language Approach to one that synthesizes reading instruction based on explicit lessons in key competence areas: synthetic phonics and daily instruction that offers learners opportunities to practice reading, writing, speaking and listening in the familiar language. It is believed that Instruction in the familiar language will give learners a strong foundation on which to build literacy and language skills in English later. The intention is to provide an effective additive bilingual program where literacy skills acquired in familiar languages support the acquisition of literacy in English while at the same time sustaining and strengthening literacy in familiar 
languages. The document was designed for all those involved in the development of literacy for early childhood education and primary schools in Zambia. Authors of early childhood education and lower primary school books will use the framework to develop materials in literacy courses. It is also, anticipated that teachers will share this approach to teaching literacy with parents and other community stakeholders. The road to reading begins early in life with parents as partners in education. Children develop early language skills from home and continue to build upon them as they enter school. The document gathers together the underlying foundation of literacy instruction from homes to classrooms; pre-school to Grade 7 and to different levels in case of children with intellectual disability.

While access to schooling in low-income countries has increased dramatically following the Education For All (EFA) campaign and the establishment of the Sustainable Development Goals (SDG) goals, evidence indicates that student achievement is very low (World Bank, 2006). Results from Early Grade Reading Assessments (EGRA) in low-income countries show that presently, very few children can read at early grade level, this includes learners with intellectual disabilities. The Zambia National Educational cooperation (ZANEC) 2010 report revealed that the literacy levels are very low among the Zambian population. Reading is a foundational skill for learning across subject areas. Children who learn to read can then read to learn content subjects. They can become independent learners for life. However, if children are not successful in learning to read in the early grades, they fall further behind and are likely to drop out of school,(Tambulukan and Bus, 2011).

Unlike learning to speak a language, learning to read is not a naturally-developing skill; it requires an adaptation of the brain to be able to recognize letters and words (Wolf, 2007). Carefully planned instruction is necessary; reading must be taught as a subject in schools; and time on task is essential if learners are to develop the cognitive processes to become fluent readers. Scholars in reading acquisition in multiple languages (Chiappe et al., 2002; Sprenger-Charolles, 2004); Adadzi, 2006; Linan-Thompson and Vaughn, 2007), have found that learning to decode print - that is, breaking apart or "sounding out" written words into letter sounds - can be done in almost any alphabetic language and requires five key skills, which have been endorsed by the US National Reading Panel (2000).

The emphasis on "early" in reading instruction is key; evidence indicates that if strong foundational reading skills are not acquired early, the gaps in reading ability and achievement grow larger over time. Stanovich (1986) called this the "Matthew Effect" in learning to read. This is a biblical concept over non achievers. Children who fall behind in learning to read typically become entangled in a cycle of failure and among such children are learners with intellectual disabilities. Low performing readers read less, and as a consequence, they do not gain vocabulary, background knowledge, and information about how reading material is structured. Children below a certain level of literacy competences by the end of Grade 1 are more likely to remain behind. If they cannot read, they are more likely to fall behind in all other subjects, repeat grades, or drop out. Therefore, it is important that all children with and without disabilities learn to read within the first few years of schooling; once they learn foundational skills, they can focus on more complex comprehension strategies and interactions with texts across subjects (Gove and Cvelich, 2011).

For many children in primary school, the language of instruction has traditionally been a different language from the one spoken in their homes. However, research shows that children learn best in their mother tongue, with a gradual transition to bilingual education. Moreover, research shows children's ability to learn a second language (e.g., an international language) does not suffer by first learning to read in their mother tongue; literacy in one's mother tongue develops the foundational cognitive and linguistic structures for learning additional languages more easily (Kosenen, 2005; UNESCO, 2011).

The teacher's role in the delivery of quality and effective instruction in literacy is critical to the success of this literacy program. It goes without saying therefore, that student teachers in Colleges of Education and Universities need to be equipped with knowledge and skills necessary to teach literacy in early childhood education and lower primary schools. This preparation should be strengthened through school-based Continuous Professional Development (CPD).

The success of any literacy program also depends on the availability of suitable reading materials to sustain and reinforce literacy skills. There is need to revamp libraries to make available reading 
materials. Schools should in addition endeavor to inculcate the culture of caring for and maintaining a wide variety of reading materials. Communities should also be called upon to help generate reading materials based on oral traditions. This will enhance access to a variety of reading materials for learners to interact with. The experience learning approach will bridge the gap between what children are familiar with at home and what they interact with at school. This enables learners to make sense of what they learn.

One of the challenges and threats to literacy instruction is that contact time between teachers and learners is not sufficient. Time on task - the amount of time teachers and learners are engaged in learning - is reduced due to poor lesson planning and absenteeism. It is, therefore, important that much time in the early grades should be dedicated to literacy instruction. Planning for extra-curricular activities that engage learners in reading practice is an additional solution to providing extra learning time more especially to learners with intellectual disabilities. Learners with intellectual disabilities are characterized by general delays in cognitive development that influence the acquisition of language and academic skills. They learn more slowly than the typical children, (Micheal \& Roseburg etal, 2011:209).

The classroom environment in many schools provides limited opportunities to support literacy. In the classroom, the absence of desks, boards and storage space can hamper literacy development. School management teams are encouraged to develop initiatives that will provide the basic requirements important for teaching literacy.

The use of English as a medium of instruction in schools especially in the early years has been disadvantageous for the majority of learners. This has not only contributed to poor acquisition of literacy skills but also mediocre academic achievement. Reading instructions thrives of the learner's knowledge of a language's sound system, vocabulary and structure.

\subsection{Literacy Situation in Zambia}

Many research findings support the opinion that Zambian children are not gaining basic literacy skills. The baseline study of the Zambian Primary Reading Program conducted in 1999 noted that among grade 1-6 learners that were tested, the majority of children that attempted to read, read at two grades below grade level in English and three grades below grade level in their own Zambian language. The Grade 5 National Assessment Survey for 2006 and 2008 reflected learning achievements below 40\% in both English and Zambian Languages (35.3\% and 39.4\% respectively) and this percentage has been stagnating since 1999. The Grade 5 National Assessment Survey and the EGRA survey, both from 2010 have shown poor reading and writing abilities among learners including learners with disabilities. The Southern Africa Consortium for Monitoring Education Quality (SACMEQ III) of 2010 noted that among Grade 6 learners that were tested in reading, only $27.4 \%$ were able to read at a basic competency level. This is clearly a wakeup call for the Ministry of Education to realize that drastic measures need to be put in place to create an environment necessary to increase learner achievement in literacy. This is what this Early Reading Program (ERP) seeks to address. It proposes some strategies aimed at redressing weaknesses that have been identified in the existing systems. Chief among these have been:

- Inadequate pre-service training in literacy instruction

- A curriculum not sufficiently focused on literacy instruction

- Inadequate provision of in-service training in literacy teaching methodology

- Half-hearted attempts at developing primary language skills and literacy

- Lack of parental or community participation and support for literacy instruction

- Inadequate provision of appropriate reading materials

- The absence of a reading culture in the country which can be blamed on the use of English in early stages of education that impedes the development of fluency in reading. Failure to read fluently frustrates attempts at reading widely with enjoyment and hence the poor reading culture.

It is in light of the low achievement results that the Ministry of Education has taken steps to improve the situation. According to the National Policy on Education, "Educating Our Future," (1996:34) "A fundamental aim of the curriculum for lower and middle basic classes is to enable pupils to read and 
write clearly, correctly and confidently, in a Zambian language and in English and to acquire basic numeracy and problem solving skills. However learning to read and write in English should begin after learners have acquired basic skills in the familiar language."

Low literacy levels in primary schools can be solved by scaffolding learning through instructions in familiar languages. This, together with a well-defined literacy teaching program will improve results. The rationale for teaching in a familiar language is rooted in scientific research which supports developing a learner's language abilities - vocabulary, intrinsic knowledge of grammar rules, and use of his or her language, in order to develop reading and writing skills. The speed and ease at which a learner can do this in his own language far surpasses that at which a learner can in a foreign language. Additionally, learning to read hinges upon a child's confidence to learn and understand. There are far more opportunities to do so in a familiar language. The composite knowledge and skills of how one reads are then available to learners to use when learning new languages, more with learners with intellectual disabilities.

While many factors affect education quality, the language of classroom instruction fundamentally impacts on a child's ability to read and learn. "This is because learning in one's first language is 'essential for the initial teaching of reading.' Children with and without intellectual disabilities, arrive on the first day of school with thousands of oral vocabulary words and fact knowledge of the sound system of their familiar language, but are unable to use and build upon these linguistic skills because they are instructed in a foreign language. This change of instruction adversely affects learners with intellectual disabilities ability to learn to read. Dismissing this prior knowledge, and trying to teach children to read in a language they are not accustomed to hearing or speaking, makes the teaching of reading difficult, especially in under-resourced schools in developing countries like Zambia.

\subsection{Teacher Competencies}

In order for teachers to teach effectively to learners with mild intellectual disabilities in the lower grades, teachers need to be competent in the language use for the children they are teaching. However this does not mean that in order for one to teach in a particular language for instance, Bemba he/she should be Bemba by origin but rather the teacher should have gained some language competencies that will enable him to teach with less challenges concerning language use. One important factor to consider is the language appropriateness, using language appropriate to the level of learners. This will make the learners to gain interest to whatever they are learning as that will enhance comprehension of what is being taught in and outside class, (Muzumara, 2011:24).

\subsubsection{Parental Involvement Affects Student Achievement}

While student success largely relies on factors like study habits, school attendance, test-taking abilities and more, research is showing that parental involvement also has a large impact on student achievement in the classroom. In fact, according to the Academic Development Institute in the USA, parents who get involved with their children's education tend to demonstrate good parenting skills, volunteer their time in the school, communicate with faculty and take an active role in school-related decision-making. This involvement provides the much needed scaffolding in supplementing the teacher's efforts at home.

The Harvard Family Research Project of (2015) concluded that parental involvement is associated with higher student achievement. This could be more beneficial to children with disabilities since they require more teaching in order to internalise what they learn. They found that student success was higher in a variety of areas including standardized test scores, grades and teacher ratings. Students are also more likely to enrol in higher-level programs, pass their classes, attend school regularly, and have better social skills and move onto post-secondary education. The types of parental involvement that had the greatest impact on student success were reading and communicating with one's child, as well as holding parental expectations. Children with intellectual disabilities stand to benefit more in the learning process when there is full parental involvement in their learning. The use of familiar language makes the acquisition of concept explicit and easy to learn. Catherine, et al, (2005), adds that evidence suggests that an encouraging home- literacy environment has a positive relationship with early literacy. Researchers have advocated joint parent preschooler book reading, believing it to be beneficial for preparing children with intellectual disabilities for formal reading instructions. This 
Use of Familiar Language to Enhance Reading Skills in Learners with Mild Intellectual Disabilities in Early Grades Primary Schools in Southern Province of Zambia

implies that the stories parents and peers tell to young children create a rich foundation for reading and this can be effective if parents take an active role in preparing their children with intellectual disabilities for future reading.

\subsubsection{How to Get Parents Involved in Education}

Communicate regularly; it is important to keep a clear line of communication with parents regarding their child's progress. If parents don't know how their child is doing, they may be less likely to get involved. According to research evidence, teachers who regularly communicate with parents generally see a positive effect on students' test scores. Send home weekly newsletters with the students that share information regarding classroom success, what the learners are working on and contact information for teachers and the school. Parents to such learners have taken an active role in the education of their children and that children have made progress in their academic achievement. Provide voluntary opportunities to the parents as a way to make the get involved in the learning of their children. This could range from offering parents the opportunity to come visit their child's school to finding ways to get them to help out, whether in the classroom or on a school field trip. Give the children interactive home work assignments. Create homework assignments that are going to bring parent and child together. This could involve a worksheet asking parents to describe their child's work or to share their understanding of educational concepts. According to the Centre for Public Education, teachers who involve parents in school work saw a boost in student scores in both writing and science. Teachers and parents alike are seeing success with blended learning approaches that can be incorporated both in and out of the classroom through the use of familiar language. Encourage parents to create a home environment that promotes learning to their children with intellectual disabilities and reinforce what is being taught at school by the teachers. This may help students with intellectual disabilities to foster the life skills they need to carry with them outside of the classroom.

\subsubsection{Parental Involvement}

As the culture of reading is strengthened within schools and learners make strides in their familiar language, which they speak at home, USAID/Read to Succeed is reaching out to parents and communities to build support for literacy education after the school day ends and keep learners on course for reading success. Across the six target provinces in Zambia, USAID/Read to Succeed schools have established 1,204 School Community Partnership Committees to engage community members in their children's education and key decisions in school management. The development of education system nowadays is not only focused on the role of teachers, but also requires involvement of parents and communities to help the schools. This is because parents especially can better understand the school system and the development of their children thus avoiding them from getting confused about assessment. However, the pattern of parental involvement should change from traditional to more effective participation through collaboration (Friend \& Cook, 2003). According Maryln (2004), collaboration refers to the style or approach in the interaction between schools, parents and community based on the elements of voluntarism, equality accomplishment of common/shared goals, sharing of responsibility in making decisions, sharing of results, sharing of resources and finally having mutual trust and respect to each other . Collaboration has positive implications on learner's academic achievement. Many learners, with and without intellectual disabilities have been successful in academics/schools and have stable emotional and social development because of the collaboration between schools and parents and other stakeholders. However, there are still some who are not willing to accept parental involvement in school and therefore try to prevent that from happening. The importance of collaboration has been acknowledged by past research (Mapp, 2002). Collaboration program can be implemented in all schools including Special Schools which often have to deal with costly services and also in need of the services from other professionals such as psychologists, speech therapists etc. Special schools are increasing and so is the number of students with disabilities, this poses great challenges to the schools as they have to deal with the lack of specific knowledge and skills dealing with special students, shortage of teaching experts and the high cost of providing services to such students. Barth (1990) proposed a collaboration program as a fundamental group activity that can help to accomplish goals of various organizations. Therefore, schools that strive to succeed must be able to solve problems by using the strategy of approaching the students parents and community in order to understand the different cultural, socioeconomic, ecological experiences among them (Patricia \& Patrick, 2007). The schools 
must also be willing to cooperate with other parties through collaboration. The schools are key actors in the implementation of collaboration programs. Thus it is necessary for school administrators to act wisely in planning strategies to encourage the involvement of parents and community. The teaching of learners with intellectual disability cannot be effective without the involvement of other stake holders such as parents to the children and other experts. The schools should establish a close relationship with parents and the community because according to Harry et al (1995), good rapport is the basis of collaboration. Schools should reflect on the extent of which they have implemented the process of collaboration. Apart from that, schools should explore and master the skills and knowledge to win the hearts of parents and communities so that they are willing to cooperate with the schools. Needs and expectations of parents have to be understood to achieve more effective communication and interaction. It is assumed that the introduction of familiar language to teach children with intellectual disabilities in lower grades might enhance parental involvement in the education of their disabled children. In this study, i consider a classroom, school environment and family as places or space for pedagogical encounters in wherever singularity. School classroom and home environment if well structured and planned to inculcate literacy skills, has the potential to transform the reading levels among learners with intellectual disabilities positively. Learners with intellectual disabilities can integrate their cultural experiences into what they learn in the classroom with others to improve their reading abilities. Therefore the engagement of the family and society through the use of familiar language to teach learners with intellectual disabilities can be an effective strategy to enhance reading skills to the learners.

\subsection{Teaching Strategies}

To fully address the limitations in intellectual functioning and adaptive behavior often experienced by individuals with mild intellectual disabilities, teachers need to provide direct instruction in a number of skill areas outside of the general curriculum. Browder, Courtade, Gibb and Flower, (2008) states that recent research is demonstrating that with an integrated and systematic approach, students with intellectual disabilities can successfully combine the separate skills of phonemic awareness and letter sound correspondence to decode unfamiliar words. The integrated approach also resonates precisely with mult component intervention approach by Cheatham, et al., (2010) and Roberts and Jones et al (2010), which has proved to be more effective in the teaching of reading to children with intellectual disabilities unlike the traditional sight word instruction. These skills are more functional in nature but are absolutely essential for the future independence of the individual. Additional skill areas include money concepts, time concepts, independent living skills, self-care and hygiene, community access, leisure activities, and vocational training. Students with intellectual disabilities learn these skills most effectively in the settings or activities in which they will be asked to apply these skills. Once the skills are mastered, then additional environments can be added to work towards generalization. This is in line with Vygotsky`s social cultural theory, stating that learning through the interface of the social cultural experience of an individual. Children learn better and faster through social interactions in ones social culture.

Muzumara (2011) noted that teachers have to be imaginative, selective and willing to adapt and keep on improving on their teaching strategies to accommodate a wide range of pupils abilities and language limitations. Teaching through a variety of practical activities and use of concrete examples, maximizes learning. Pupils with limited language skills and those with intellectual disabilities may find learning easy and interesting if they are involved in activity-based learning.

Teachers need to identify barriers associated with language so that they can employ appropriate teaching strategies in class. Effective use of familiar language by the teacher is likely to yield a myriad of results in reading skills by the learners with intellectual disabilities. The use of computer games which associate Sounds to Letters and Objects Identification (SLOI) such as the graphor game can help to create letter knowledge awareness and phonological awareness. These are key structures in the enhancement of reading skills in learners with mild intellectual disabilities. Adams, (1990), states that "phonological awareness is ones awareness of and access to the sound structure of oral language which helps an individual to manipulate individual sounds in words. Therefore the emphasis to educators is to ensure that children reach a desirable level of letter knowledge and phonological awareness, only then can they make progress in their reading abilities. Gombert, (2002) suggests that providing phonemic awareness instructions in early grades is vital for the reading development of 
children with intellectual disabilities because they do not easily develop phonemic skills. The sound letter knowledge and object identification, (SLOI) approach will stimulate the sense of hearing, when a child pays attention to the sound, at the same time the child will use his sight to look at the letter associated to the sound head and also identify the picture of an object associated to that sound and the letter. One aspect of a letter and sound brings together so many senses to focus on one aspect and this has a likely hood of higher mastery level of reading. This approach can strongly be reinforced and made effective through the use of familiar language to a child with intellectual disability. The child will also use the language experience approach to recognize some pictures of objects which eventually improves comprehension of what has been head. Previously, most educators in literacy concentrated on letter sound knowledge (LSK), which required a child to master the letter first and then the sound it makes, which was more mechanical than reality based. Furthermore, due to its mechanical effect, most children just knew how to sing the alphabet and not relating sounds to letters and real objects. Therefore I strongly feel and recommend that instead of letter sound knowledge (LSK), it should be sound letter object identification (SLOI), presented in a familiar language and in later grades used to teach in English or second language.

General curriculum areas should not be neglected however, and there are some promising practices to help support these students with intellectual disabilities in a number of academic areas. One effective early literacy strategy with learners with intellectual disabilities is paralinguistic milieu teaching (Fey, et.al, 2006), a technique that ties instruction to the specific interests and abilities of the individual child. This language acquisition instructional strategy also helps support effective self-determination, as a key component of the training is frequent requesting behavior from the student.

Breaking down larger tasks into their specific component parts (task analysis) can be an effective technique for teaching any number of skills to students with intellectual disabilities. More complex concepts or activities can then be taught over time, and as the student masters' one component of the task, another is added to the routine. This type of task analysis can be taught using a variety of instructional supports, from physical and verbal prompting to observational learning. As always, the specific instructional strategies and materials used with the student should be aligned to the student's own interests and strengths.

Useful strategies for teaching students with intellectual disabilities include, but are not limited to, the following techniques:

- Teach one concept or activity component at a time

- Teach one step at a time to help support memorization and sequencing

- Teach students in small groups, or one-on-one, if possible

- Always provide multiple opportunities to practice skills in a number of different settings

- Use physical and verbal prompting to guide correct responses, and provide specific verbal praise to reinforce these responses

\subsection{Strategies that Could be used to Teach Reading}

Matafwali (2005) M.Ed thesis, states that the motion in line with Piaget's (1967), development sequence theory which postulates that, concepts developed in children progress along a continuum from concrete thinking to abstract thought. This implies that pupils can learn better through the involvement of visual, auditory and tactile senses in their learning process. Head-Taylor (1987), states that "early exposure to skills like reading and that's providing a good foundation for learning." Bannatyre, (1971), stresses the importance of basic information received through senses, he affirms to what Piaget (1967) in Matafwali (2005), stated on children's learning through concrete method of teaching as an effective way to teach pupils with intellectual disabilities. Through concrete teaching, pupils can perceive the environment through several senses, through integration of information which might be very familiar as it will be presented in a familiar language to the child. Pupils with language problems associated with intellectual disability can be taught the language skills so that they can be able to articulate properly and be able to read. By language, the child thinks and associates experiences with meaning, (Pringle and Varn, 1974:90).

Every child is capable of learning provided that teaching is appropriate to the child's level of comprehension. This means that a structured approach is necessary to the pupils, this structure 
understandably prefers to rely on a less formal or more intuitive approach to their normal pupils, parents have considerable expertise that needs to be mobilized in co-operation with specialists so that a jointly agreed program may be evolved, Wookey and Herbert, (1998). Robert (1975:132), states that "the idea that intelligence is not fixed and immutable is tremendously exciting for all working with young children. It means that you and I are not victims of the child's intelligence; we are in the business of creating it." It is the teacher and other stake holders to make strategies and ensure that the child learn regardless the disability. Robert (1975) stipulates that children should be provided with opportunities so that they can acquire some knowledge through experience that's using the familiar language creates an interface of what is being learnt to what is socially known by the child; however, deprivation of such important needs turns children with intellectual disability into deviant behavior because they might not make sense from what they are being taught. Experiences of some aspects of life can make children learn something and more especially the provision of some basic needs of a child; satisfied needs are a basis of better learning.

\subsubsection{Recommended Strategies for Teaching Reading Skills}

Torgesen (2002) points out that the fact that reading comprehension is the joint product of language comprehension ability and word identification skills must be considered when setting goals for the reading attainment of all children. In other words teachers must recognize the fact that general cognitive ability (specifically verbal ability and knowledge) strongly influences reading comprehension. Therefore the ultimate goal of reading instruction to the intellectually disabled pupils is to help them acquire the knowledge and skills necessary to comprehend printed material, at a level that is consistent with their general verbal ability or language comprehension skills.

Difficulties in teaching reading to intellectually disabled pupils are mainly characterized by late exposure to reading instructions in a familiar language. Children with intellectual abilities who are destined to be poor readers in lower grade levels almost invariably have difficulties understanding and applying the alphabetic principle in deciphering unfamiliar words. Their difficulties developing good analytic strategies for identifying unknown words make it difficult for them to read independently, and it also produces far too many word reading errors. Both of these latter consequences of failure to acquire good phonemic decoding skills affect the development of fluent word reading ability, which depends heavily on learning to identify large numbers of word by sight (Torgesen, Rashatte and Alexander, 2004).

Discovery of the core phonological problems associated with early reading difficulties has at least one unanticipated consequence. The ability to assess these core language problems directly has led to the discovery that the early word reading difficulties of children with relatively low general intelligence and verbal ability are associated with the same factors that interfere with early reading growth in children who have general intelligence in the average range (Fletcher, 1994).

Instructions for children with intellectual disabilities must be more intensive than for other children. The fact that instructions must be more explicit and comprehensive for those children implies that more skills and knowledge must be directly taught, which logically requires a greater number of teaching/learning opportunities per day than typical classroom instruction. The more they learn; they become aware of the phonological skills and consequently are able to learn to read. Reading skills should be developed and practiced using real books, real instruction cards, real receipts, real brochures and real comic books, as well as through graded readers, games and flash cards. As far as possible the 4R Test should be applied when selecting curriculum content. Intellectually disabled pupils learn well through the use of real life situations and this explains the reason why the implementation of the Sound Letter Object Identification (SLOI) concept can be very effective. This aspect makes the teaching of reading more meaningful and reduces on challenges that children encounter when taught things they are not familiar to, for instance phonemes, consonants and the letter sound knowledge. Allor, Mathes, Roberts, Cheatham and Al Otaib, (2014) studies revealed that the most effective teaching strategies for struggling children to read, have also proved to be the most effective for the population with mild intellectual disabilities. However emphasis is placed on extended period of instruction and continuous monitoring in order to ascertain the effectiveness of the intervention strategy. 
However, Carmine et al (2004) and Turnbull, (2007) in Westwood (2007:25), suggests that "in addition to reality-based learning, children with intellectual disability also need some high-quality direct teaching, with the content broken down into very simple steps to ensure high success. The most fundamental job of the nation's education system is to teach children to read (Curriculum Frame Work, 2013). In spite of this manifesto, teaching children with intellectual disabilities (ID) to read has been largely ignored in the national rhetoric. Typically it has been assumed that reading is a skill beyond the intellectual capabilities of most learners with ID and that at best they might be taught to recognize a limited number of sight words (i.e., high frequency words). As such, four in five children with mild to moderate ID never achieve even minimal levels of literacy (Katims, 2001). Recent research supports the view that this group of learners should be taught to read in a manner similar to other learners who struggle to learn to read (Mathes, Roberts, Jones, et al., 2010; Browder et al., 2008). Specifically, students with ID should be taught to read using strategies and techniques that will provide them with the skills necessary to fully process individual words in connected text and derive meaning from the text and this can be enhanced by the use of the familiar language of the child by both the logographic route and the alphabetical approach to teaching reading, the two approaches will complement each other and minimize their weaknesses. These findings suggest that when given scientifically based and rigorously intensive reading instruction over an extended period of time, these children respond positively, making important gains in literacy development. Instruction must be systematic and explicit, including all reading components; repetitive in its use of routines and instructional language; fast paced; and highly motivating. Lessons should be fully implemented by teachers skilled in effective reading instruction. Learners with mild intellectual disabilities require large amounts of repetition to make meaningful progress, (Al Otaib, et al., 2014).

\subsubsection{Summary}

Both local and international literature was reviewed in order to establish what other scholars have studied on the reading levels of learners globally and locally. This enabled the researcher to find gaps that the study can fill. Therefore, with evidence from the revealed literature it is quite clear that the use of familiar language to teach literacy has a positive impact on the learners. However it is also evident from the consulted literature that most research has been done on the "normal learners" and not on the learners with intellectual disabilities. So the effect of using familiar language to teach reading to learner with intellectual disability is not known. Learners with intellectual disabilities have serious deficits in reading. The available literature also revealed some possible mitigation measures that could ameliorate the reading challenges of learners with intellectual disabilities. The literature reviewed in this study, pointed to the fact that the collaboration between professionals and parent in the education of learners with intellectual disabilities is cardinal to the effective teaching of reading.

\section{MeTHOdOLOGY}

\subsection{Methodology and Methods}

Methodology is a philosophical assumption concerned with knowledge creation (McGregor \& Murname, 2010:420), on which researchers base their methods. However a method is an interface between a paradigm and a method. This includes the research design, target population, sample size and sampling procedures. It also looks at the data collection methods and instruments to be used, analysis of data, and limitations of the study

\subsubsection{Research Design}

This study adopted both qualitative and quantitative research design. The reason for triangulation to this study is to reduce on the limitations of a single research design, for instance quantitative research imposes restrictions on the scope of the investigation (Hebert, 1990), and this is because it requires details that can be measured in order to produce results which could be measured in order to produce results that could be generalized. However this is done not to demerit the efficacy of a single research design but rather to give strength to the research and to give in-depth understanding, which a single approach cannot attain. This is a longitudinal study that compared the reading performance of learners with mild intellectual disabilities from one academic year to the next academic year of their schooling. First data collection was between January to March, 2018 and the second phase was from January to July, 2019. In the second phase, the same learners where followed for data collection. 
Use of Familiar Language to Enhance Reading Skills in Learners with Mild Intellectual Disabilities in Early Grades Primary Schools in Southern Province of Zambia

\subsubsection{Population}

Population in this context, refers to a complete set of elements (persons or objects) that possess some common characteristics defined by the sampling criteria established by the researcher, (Dominik, \& Stephen, 2013).The target population are learners with mild intellectual disabilities, teachers teaching learners with intellectual disabilities in the selected school of Southern province, head teachers of the selected schools and parents or guardians to children. The same population was followed in the second phase.

\subsubsection{Sample Size}

Sample size refers to the number of items to e selected from the universe to constitute a sample. White (2005) defines a sample as a group or situation selected from a larger population. This means sampling is the selection of an aggregate or totality on the basis of which judgment or inference about the aggregate or totality is made. In other words, it is the processes of obtaining information about the entire population by examining only a part of it. The sample size was determined by the use of a formula by Slovene, which states that; $\mathbf{n}=\mathbf{N} / \mathbf{I}+\mathbf{N e}^{2}$. n, represents the sample size, "N" represents population size while "e" is the margin of error. Population size simply mean the number of people that fit your demographic, this number can be unknown or approximated. The margin of error or confidence interval implies that how much higher or lower than the population mean you are willing to let your sample mean fall. The margin of error is the percentage difference to make a whole or 1 , for instance the margin of error for $95 \%$ is 0.5 . The most common confidence levels used are $90 \%$, 95\% and 99\% confident. Slovenes formula is most used when nothing much is known on the effects of the variable to the population. Below is the computation to determine the sample size; the estimated population is 139 ; the confidence level is $95 \%$, giving us the margin error of $0.05 \%$. Therefore we key in the figures in the formula as follows;

\section{n- Sample size}

\section{N- Population=139}

\section{Confidence level $=95$}

E- Margin of error $=(\mathbf{0 . 0 5})^{2}$

$\mathrm{n}=\mathrm{N} / 1+\mathrm{Ne}^{2}$

$\mathrm{n}=139 / 1+139(0.05)^{2}$

$\mathrm{n}=139 / 1+(0.0025) 139$

$\mathrm{n}=139 / 1.3475$

$\mathrm{n}=\mathbf{1 0 3}$

The sample size was 103, which included 73 learners with intellectual disabilities from the selected schools, 20 teachers teaching learners with intellectual disabilities, 5 head teachers of the selected schools and 5 parents of children with intellectual disabilities from selected schools in Southern Province of Zambia.

\subsubsection{Sampling Procedure}

Singleton and Straits (2010) noted that the sampling procedures should identify a sample that is representative of the population. Meaning that the sample should closely resemble the target population on all the relevant characteristics. In this study, 73 learners with intellectual disabilities were tested on reading of some sub- test items from the BASAT in a familiar language (Chitonga) while the questionnaire was administered to 20 teachers of learners with intellectual disabilities in order to generate information on how effective the teaching of reading has been through the use of familiar language to learners with intellectual disabilities. 5 Head teachers from the selected schools were exposed to a structured interview schedule. 5 parents were also exposed to a structured interview schedule. The researcher also took time to familiarize with the learning environment under which the learners learn from and took note of the observations. The sampling technique used in this study was purposive for the schools, head teachers, classroom teachers and also the learners due to low enrolment numbers of learners with intellectual disabilities in the schools. This extreme sampling is supported by Patton, (1990), who describes this kind of sampling as extreme because significant respondents are specifically sampled because of the rich knowledge they have, that which the researchers is looking for. In other words we can say such a sample is special to some extent. 
Use of Familiar Language to Enhance Reading Skills in Learners with Mild Intellectual Disabilities in Early Grades Primary Schools in Southern Province of Zambia

\subsection{Respondent's Background Information}

The study sought to establish the demographic information of the teachers. A total of 20 teachers participated in the study of which 16 making $80 \%$ were female and 4 adding to $20 \%$ were male. The following figure below represents the bio-data for the teachers who participated in the study.

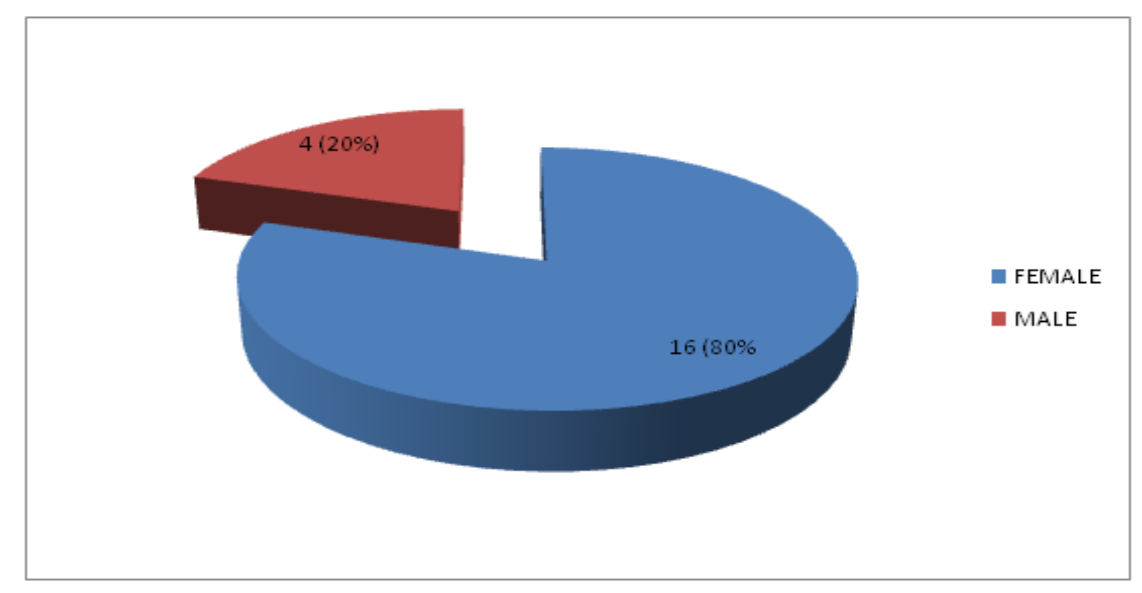

Figure1

Figure 1 represents the number of teachers who participated in the study.16 $(80 \%)$ were female while $4(20 \%)$ were male. This revelation shows that more female teachers are the ones teaching learners with intellectual disabilities in the selected schools where the study was conducted.

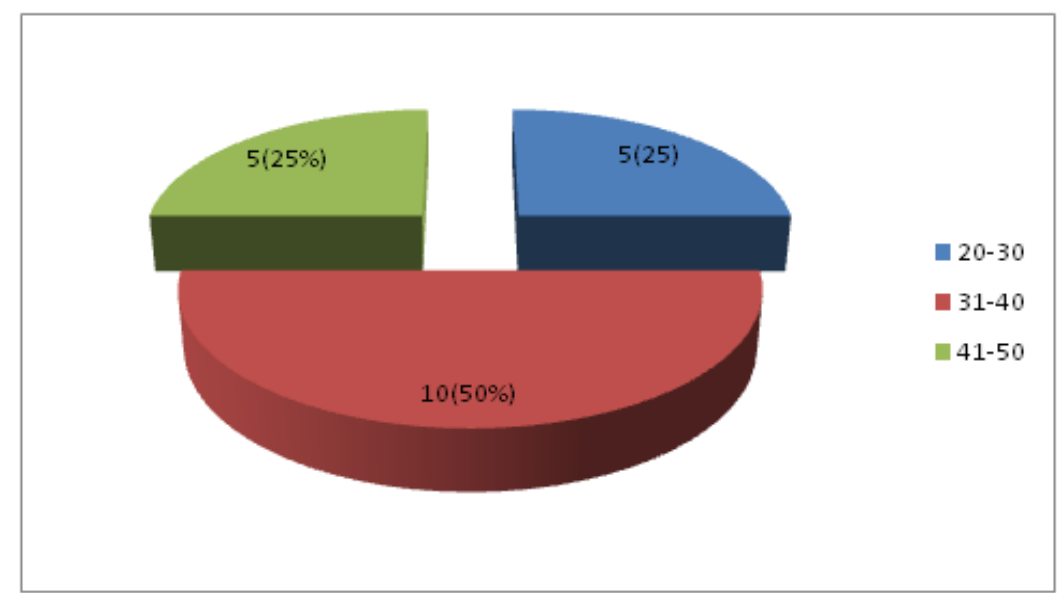

Figure2: The age range for the teachers as presented below;

Figure 2 shows that $10(50 \%)$ of the respondents age ranged from 31-40 and 5(25\%) was 20-30 years and $5(25 \%)$ to $41-50$ years respectively.

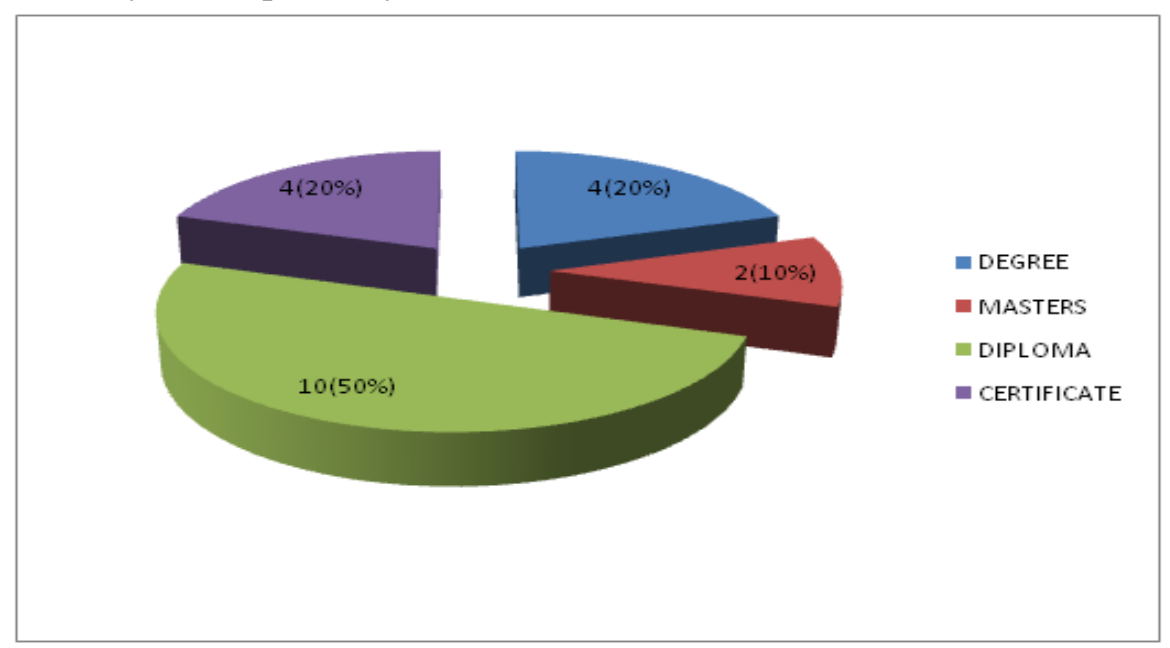

Figure3: Teachers professional qualifications 
The above figure shows the areas of professional specialization for the teachers who are teaching learners with intellectual disabilities. The area of expertise has a bearing to the teaching of children and as such the researcher thought it wise to also consider looking at the nature of qualifications for the teachers.50\% had diplomas certificates at the time of the study, $20 \%$ had degree, another $20 \%$ had certificates and $10 \%$ had masters' degrees all in special education respectively.

\subsubsection{Instruments for Data Collection}

Data collection was done in two phases and this was based on administering the questionnaires to the teachers teaching learners with intellectual disabilities, while head teachers from the selected schools shall be exposed to a structured interview schedule. Parents of children shall be subjected to a structured interview schedule. The general observation of the learning environments using the observational checklist was made in order to assess on how conducive the learning environment is to enhance reading among learners with intellectual disabilities. Pupils were tested on their reading skills by using the sub-test items in Chitonga from the BASAT. The Basic Skills Assessment Tool (BASAT) is an individual assessment tool developed by the Ministry of Education in Zambia, primarily to assess the basic reading and writing skills in the lower grades. The following reading skills were tested using this instrument, children alphabetical knowledge, phonological awareness, reading and picture comprehension. Children were assessed individually and testing took an average time of 35-45 minutes per child. Detailed description of the skills assessed is presented below:

Alphabetical Knowledge: Knowledge of the alphabet was assessed using two sub tasks: letter name knowledge and letter sound association. This was done using the printed cards of the 26 letters of the alphabet, presented to a child in a random order. The exercise was meant to ensure that children showed knowledge of the letters and not just singing the alphabet song.

Phonological Processing: Four tasks were used to assess different types of children phonological awareness skills: syllable segmentation; discrimination of initial and end sound; and blending sound.

Reading Ability: The reading test from the BASAT applied, as earlier alluded to, these reading items referred to here to, were customized in Chitonga. Children were asked to read a series of words and two sentences. The categories of worlds fell into four groups; two letter words; one syllable words; two syllable words; and three syllable words. The scores were coded on a three point scale: $0=$ to complete fail, $1=$ attempted to read; and $2=$ able to read accurately. The maximum score possible was 38 .

Reading Comprehension: The picture comprehension test from the BASAT was utilized. The test consists of four sentences with corresponding pictures. The maximum score was four (4). The reason for the triangulation of the test tasks was to get detailed information on the reading skills of the learners.

\subsubsection{Procedures for Data Collection}

The Ontological assumption influences the type of questions and data to be collected, therefore, quantitative and qualitative data was collected through administering questionnaires to teachers teaching learners with intellectual disabilities in the selected schools. Reading levels among the learners' was established through a reading test administered to learners with intellectual disabilities. Data from head teachers from the selected schools was sought through structured interview schedules and the learning environment was observed for quality assurance. An observation of the learning environment was also made in order to assess on how conducive and stimulating the learning environment was to enhance reading among the learners with intellectual disabilities. This observation also included the observation of the actual classroom, where learners learn from. To this effects field notes were taken. Mulhall, (2003), complements to the fact that field note as a research tool is very important in observational studies and ethnographies in particular. However these note take different forms as to how, when and where the researcher use them. The process of observations had its own limitations as the presence of the unfamiliar person around the school could attract a different kind of behavior from both the pupils and the teachers, hence giving a wrong impression to the researcher. The strength of the observation was that it brought the aspect of validity to the data given by the teachers because the researcher cross checked the data given by teachers and what was observed from the learning environment.

\subsubsection{Reliability}

In order to enhance reliability of the findings, the data collected was verified by using triangulation and respondent validation. Triangulation was used to compare data from questionnaires for teachers 
Use of Familiar Language to Enhance Reading Skills in Learners with Mild Intellectual Disabilities in Early Grades Primary Schools in Southern Province of Zambia

and data from the interview. To ensure that the findings are valid, the researcher cross- checked the respondent's responses with those of other respondents in relation to available literature. Further validation was also done through cross checking the data generated from observations to that of the questionnaires and interview schedules.

\subsubsection{Data Analysis}

Ader (2008:333) describes research data analysis as a "process of inspecting, cleaning, transforming, and modeling data with the goal of highlighting useful information, suggesting conclusions and supporting decision making". Quantitative data collected shall be analyzed using the Statistical Package for Social Science (IBM SPSS 25.0), while qualitative data shall be analyzed thematically. Specific research questions and themes from the structured interview schedules shall be analyzed thematically. SPSS was used in order to obtain frequencies and percentages. Further, to have valid analysis of data, inferential statistics analysis strategy was also used to systematically analyze quantitative data, based on the data collected in the two phases and their scores.

\section{LIMITATIONS OF THE STUDY}

The research was only limited to head teachers, parents, teachers teaching learners with mild intellectual disabilities in the lower grades and levels in selected schools. This research was also limited to learners with mild intellectual disabilities in the selected schools in southern Zambia.

\subsubsection{Ethical Consideration}

Ethical approval letter was sought from the University of Zambia (HSSEC) before commencement of data collection. This was done to facilitate and underpin the observance of ethical considerations. Permission was also sought from the District Education Board Secretary (DEBS) office to visit the selected schools for data collection. At the school, permission was also sought from the head teacher. The data collected was absolutely kept as secrete and for the intended purpose which is academic in other words, confidentiality of all participants was highly upheld before and after the research. Learners were not forced to take part in the study and were allowed to leave at their own discretion. Names were not included in the data collected and questions that may cause discomfort to the respondents were avoided.

\subsubsection{Summary}

A longitudinal study design was implemented in the study; both qualitative and quantitative research methods were used. Purposive sampling procedure was also used in order to generate detailed information. Qualitative data was analyzed using the statistical package for social sciences (SPSS) while qualitative data was analyzed thematically by drawing themes from the data generated. The study was delimitated in some selected schools in southern province of Zambia, the principle of confidentiality was upheld throughout the study.

\section{Presentation of Research Findings}

\section{Reading Scores for Phases 1, Familiar Language}

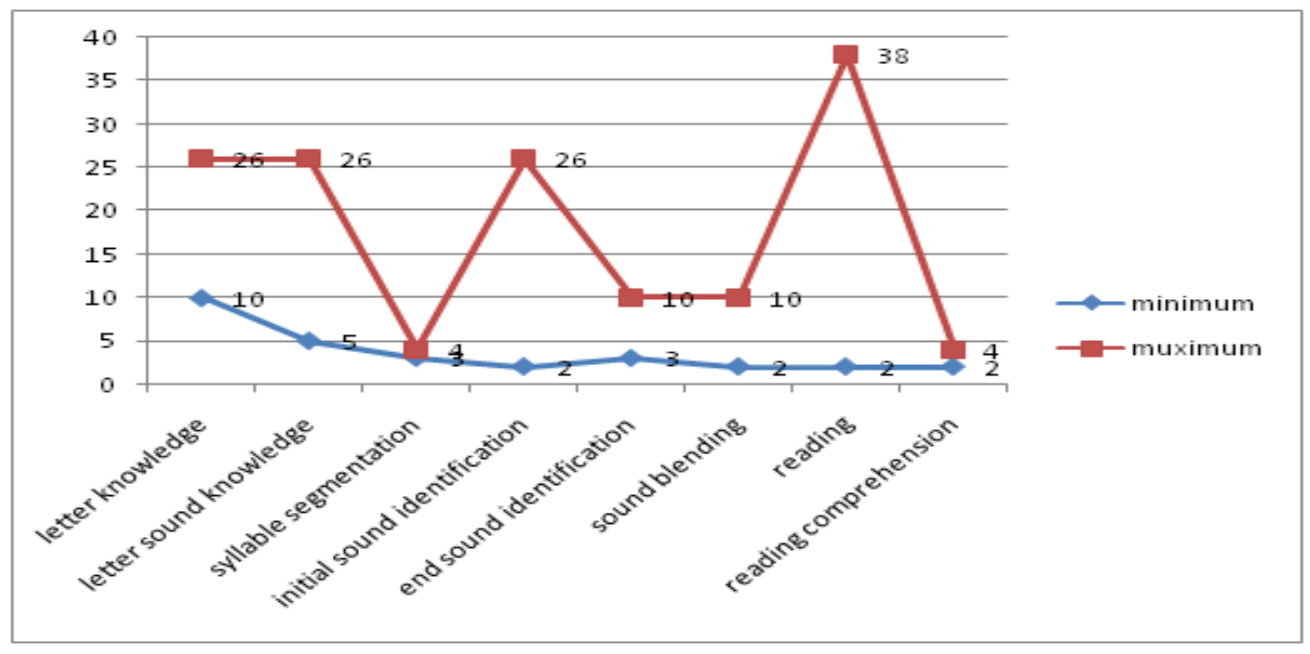

Figure4: represents the reading scores on the reading sub test from the BASAT during phase 1. 
Use of Familiar Language to Enhance Reading Skills in Learners with Mild Intellectual Disabilities in Early Grades Primary Schools in Southern Province of Zambia

As shown above, letter knowledge had a total of 26 items. Children were required to sing the alphabet song, identify, and name the letters of the alphabet. The highest score on this subtest was 10 . The other two subtests under alphabetic knowledge were identification of letter sounds, and letter sound association each of which had a total of 26 test items. The highest scores on these sub tests were 5 and 3 respectively. Further we assessed phonemic awareness through syllable segmentation, discrimination of initial and ending sounds, as well as blending the sounds into words. Syllable segmentation had a total of 4 tasks and the highest score was 3, discrimination of initial and ending sounds had a total of 10 tasks each. The highest score was 2 and 3 respectively. Blending sounds into words had a total of 5 tasks, and the highest score obtained by the participants was 2 .

The researcher further assessed reading skills using a number of test activities such as, reading name, reading two letter words, reading two syllable words, reading three syllable words, reading short sentences, and reading comprehension. The overall total was 38 scores. The highest scores on individual subtests ranged from 0-2 respectively. Performance on basic skills was relatively poor as shown by the low scores.

\section{Reading Levels of Learners in Chitonga (Familiar Language) Phase 2 as Presented Below:}

Table1

\begin{tabular}{|l|l|l|l|l|l|}
\hline \multicolumn{7}{|c|}{ Descriptive Statistics } \\
\hline & $\mathrm{N}$ & Minimum & Maximum & Mean & Std. Deviation \\
\hline LETTERKNOWLEDGE & 73 & .00 & 10.00 & 7.1806 & 7.58859 \\
\hline SOUNDKNOWLEDGE & 73 & .00 & 26.00 & 5.9167 & 7.55590 \\
\hline SYLLABLE & 73 & .00 & 10.00 & 1.2361 & 2.08612 \\
\hline INITIALSOUND & 73 & .00 & 20.00 & 3.3194 & 4.07613 \\
\hline ENDSOUND & 73 & .00 & 10.00 & 2.6528 & 3.54909 \\
\hline SOUNDBLEND & 73 & .00 & 6.00 & 1.5211 & 1.51053 \\
\hline READING & 73 & .00 & 38.00 & 9.0417 & 10.64066 \\
\hline COMPREHENSION & 73 & 2.00 & 4.00 & 3.8889 & .42972 \\
\hline
\end{tabular}

The descriptive statistics above shows the reading scores of the reading sub test of the BASAT for phase 2 of data collection. The computation was done using SPSS.

Table2: Comparisons of the Reading Scores

\begin{tabular}{|l|l|l|l|l|l|}
\hline \multicolumn{5}{|c|}{ Paired Samples Statistics } \\
\hline & & Mean & $\mathrm{N}$ & Std. Deviation & Std. Error Mean \\
\hline \multirow{2}{*}{$\begin{array}{l}\text { Pair } \\
1\end{array}$} & PREVIOUS & 3.6250 & 8 & 2.77424 & .98084 \\
\cline { 2 - 6 } & CURRENT & 4.7500 & 8 & 3.28416 & 1.16113 \\
\hline
\end{tabular}

Table3: Paired Samples Correlations

\begin{tabular}{|l|l|l|l|l|}
\hline \multicolumn{5}{|c|}{ Paired Samples Correlations } \\
\hline & & $\mathrm{N}$ & Correlation & Sig. \\
\hline Pair 1 & PREVIOUS \& CURRENT & 8 & .631 & .093 \\
\hline
\end{tabular}

Table4: Paired Sample test

\begin{tabular}{|c|c|c|c|c|c|c|c|c|c|}
\hline \multicolumn{10}{|c|}{ Paired Samples Test } \\
\hline & & \multicolumn{5}{|c|}{ Paired Differences } & \multirow[t]{3}{*}{$\mathrm{t}$} & \multirow[t]{3}{*}{ df } & \multirow{3}{*}{$\begin{array}{l}\text { Sig. } \\
(2- \\
\text { tailed) }\end{array}$} \\
\hline & & \multirow[t]{2}{*}{ Mean } & \multirow[t]{2}{*}{$\begin{array}{l}\text { Std. } \\
\text { Deviation }\end{array}$} & \multirow{2}{*}{$\begin{array}{l}\text { Std. } \\
\text { Error } \\
\text { Mean }\end{array}$} & \multicolumn{2}{|c|}{$\begin{array}{l}95 \% \text { Confidence Interval } \\
\text { of the Difference }\end{array}$} & & & \\
\hline & & & & & Lower & Upper & & & \\
\hline $\begin{array}{l}\text { Pair } \\
1\end{array}$ & $\begin{array}{l}\text { PREVIOUS } \\
\text { CURRENT }\end{array}$ & -1.1250 & 2.64237 & .93422 & $\begin{array}{l}-33408 \\
\end{array}$ & 1.08408 & $\begin{array}{l}- \\
1.204\end{array}$ & 7 & .268 \\
\hline
\end{tabular}

The table above presents the comparative statistics between the previous scores and the current scores on the reading abilities of learners with mild intellectual disabilities. The Paired Sample Test correlations statistics test was used to assess whether the medians were different across the two phases and results shows that there is no significant difference in the reading levels between the two phases. $F(1,14)=0.171$ and $P>0.05$. The difference is not significant. 
Use of Familiar Language to Enhance Reading Skills in Learners with Mild Intellectual Disabilities in Early Grades Primary Schools in Southern Province of Zambia

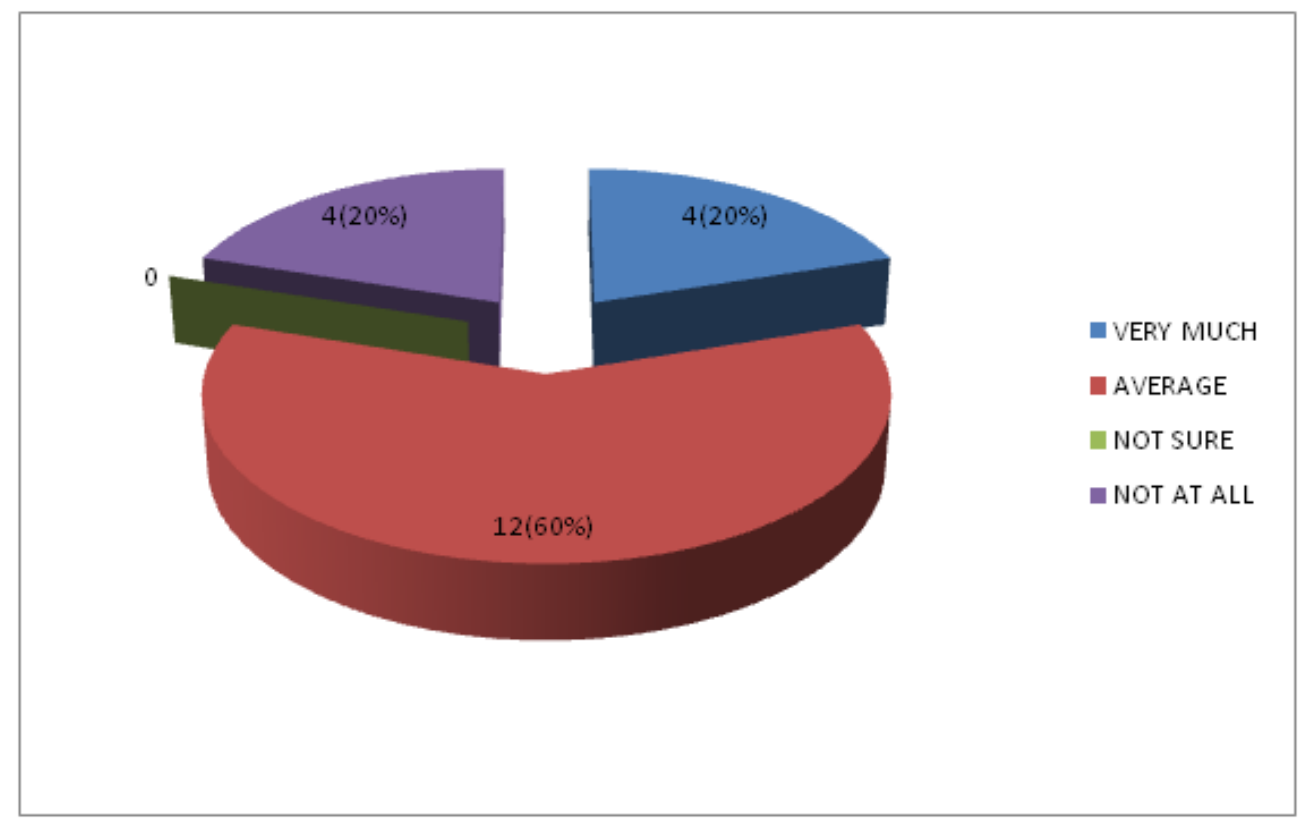

Figure5: Benefits of Using Familiar Language to Teach Reading (Teachers' Views and Observations)

The figure above presents the information on the benefits of using familiar language to teach reading to children with intellectual disabilities.60\% stated that the effectiveness was average implying that not much gains were made by the learners as a result of learning in a familiar language. $20 \%$ said that teaching in a familiar language was very effective as it made most learners to improve their reading ability. Another $20 \%$ said they were not sure whether the use of familiar language is effective in the teaching of reading to learners with intellectual disabilities. None stated that nothing at all was enhanced in terms of reading improvement among learners with intellectual disabilities.

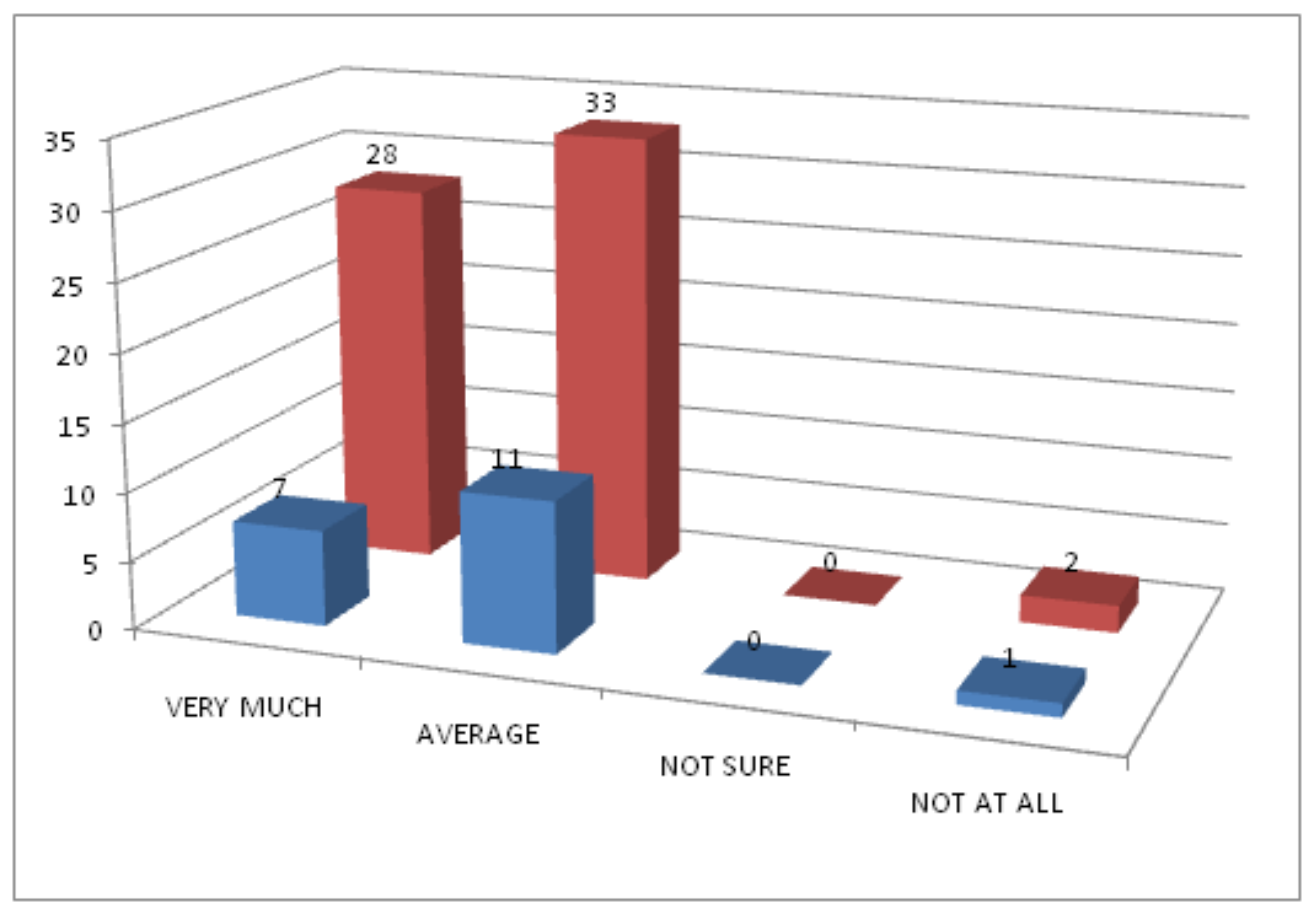

Figure6: Teachers' competence in the familiar language used to teach reading to learners with intellectual disability

The rating scale was on a 4 point scale in the following order; not at all $=1$ point, not sure $=2$ points, average $=3$ points and very much $=4$ points. Therefore 7 respondents stated that they were very conversant with the familiar language they were using to teach the learners, 11 were average while none were not sure and only 1 said was not competent in the use of the familiar language used to teach the learners. 
Use of Familiar Language to Enhance Reading Skills in Learners with Mild Intellectual Disabilities in Early Grades Primary Schools in Southern Province of Zambia

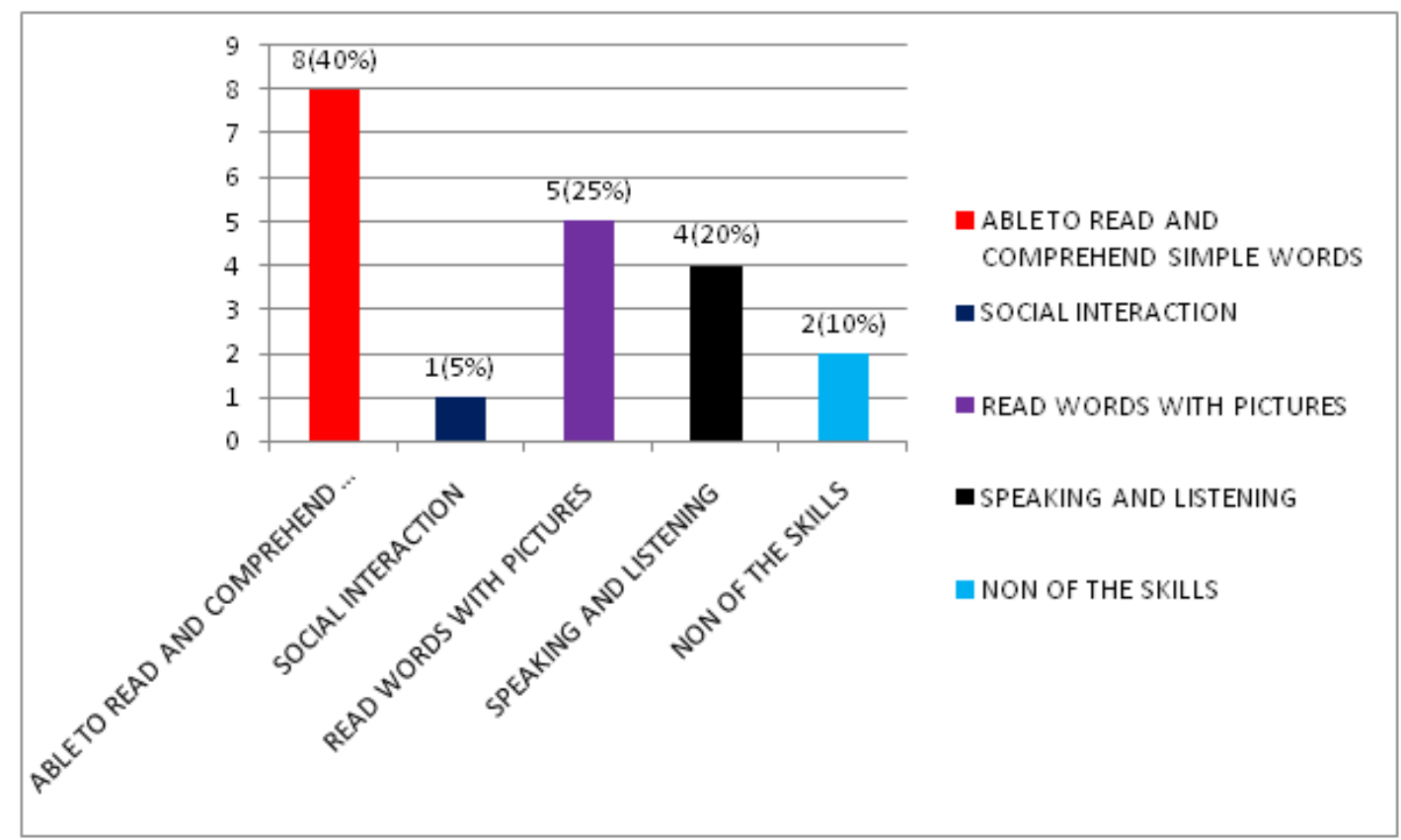

Figure7: Reading skills enhanced as a result of teaching in a familiar language

Figure 6 presents the responses from the respondents on the reading skills they have observed in the learners with intellectual disabilities over the time that they have been teaching using the familiar language. $40 \%$ of the teachers who took part in the study stated that most learners are able to read simple words and understand, $5 \%$ said the use of familiar language has enhanced social interaction and participation in class by the learners, $25 \%$ said learners are able to read words with pictures while $20 \%$ have improved the skills of speaking and listening and $10 \%$ said none of the skills have been enhanced.

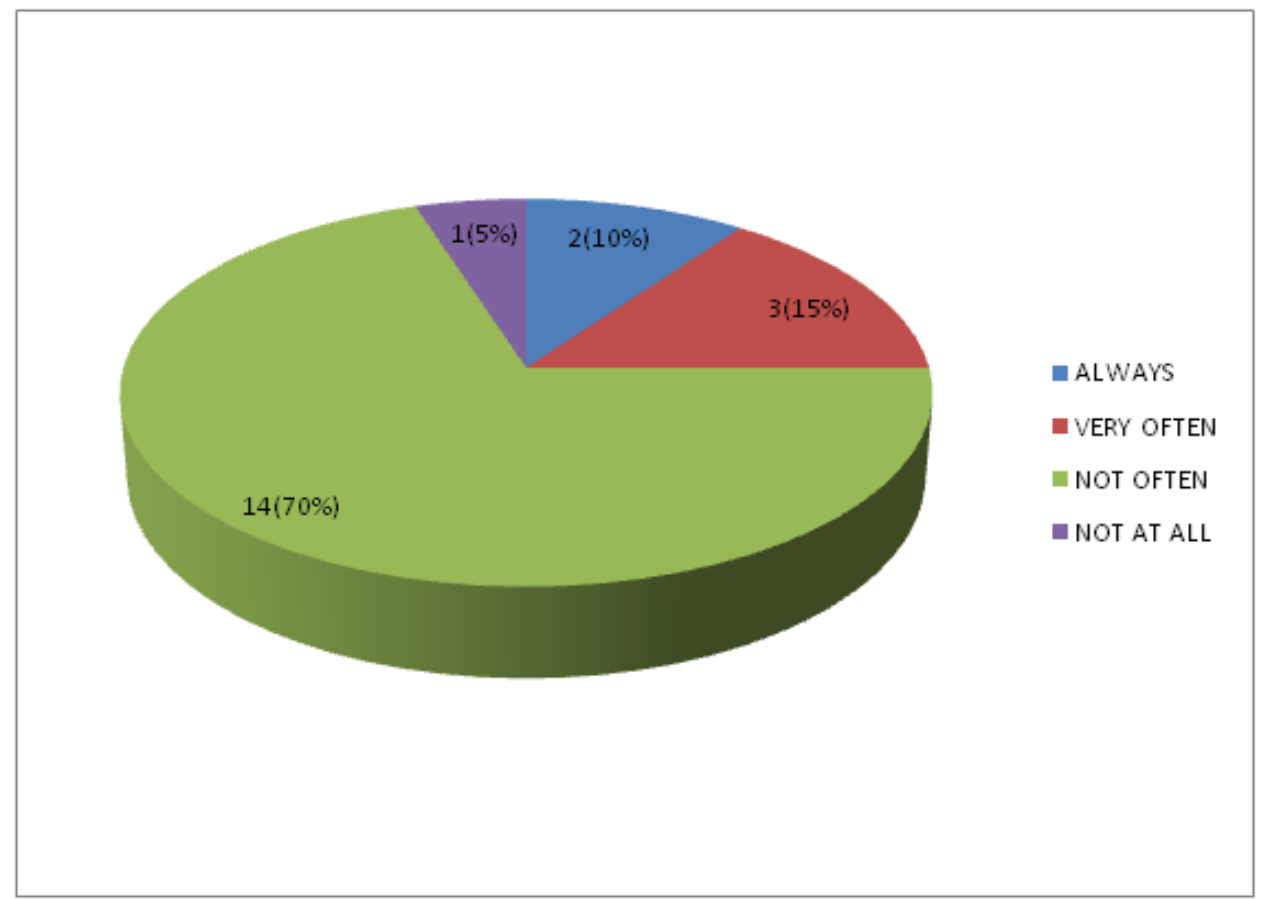

Figure8: Parental involvement as a result of teaching using familiar language

The figure above shows responses from respondents on the enhancement of parental involvement in the education of their children with intellectual disabilities as a result of the use of familiar language to teach. The majority of respondents stated that parents are not often involved the learning of their children and this amounted to $70 \%$ while $15 \%$ said very often, $10 \%$ said always parents are involved and $5 \%$ respectively said not at all. 
Use of Familiar Language to Enhance Reading Skills in Learners with Mild Intellectual Disabilities in Early Grades Primary Schools in Southern Province of Zambia

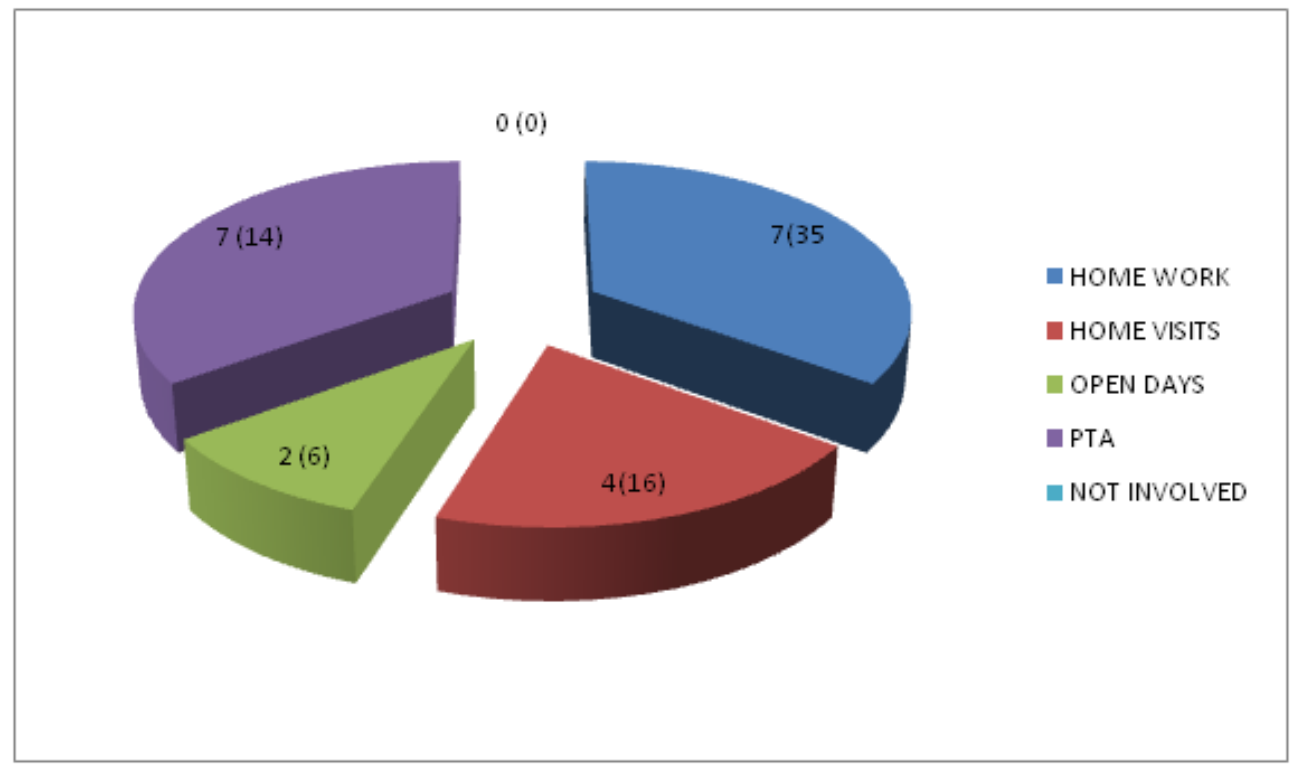

Figure9: Means by which parents are involved in the learning of their children

Figure 8 presents ways in which parents are being involved in the learning of their children with intellectual disabilities. Four (4) respondents said home visits amounting to 16 points, 7 teachers responded that parents are involved through the home work assignments give to pupils, amounting to 35 points, another 7 teachers stated that parents are engaged in the learning of their children through Parents Teachers Association (PTA) meetings, scoring 14 points. 2 stated that parents are involved through open days amounting to 6 points and non stated that they are not involved in any way, giving 0 .

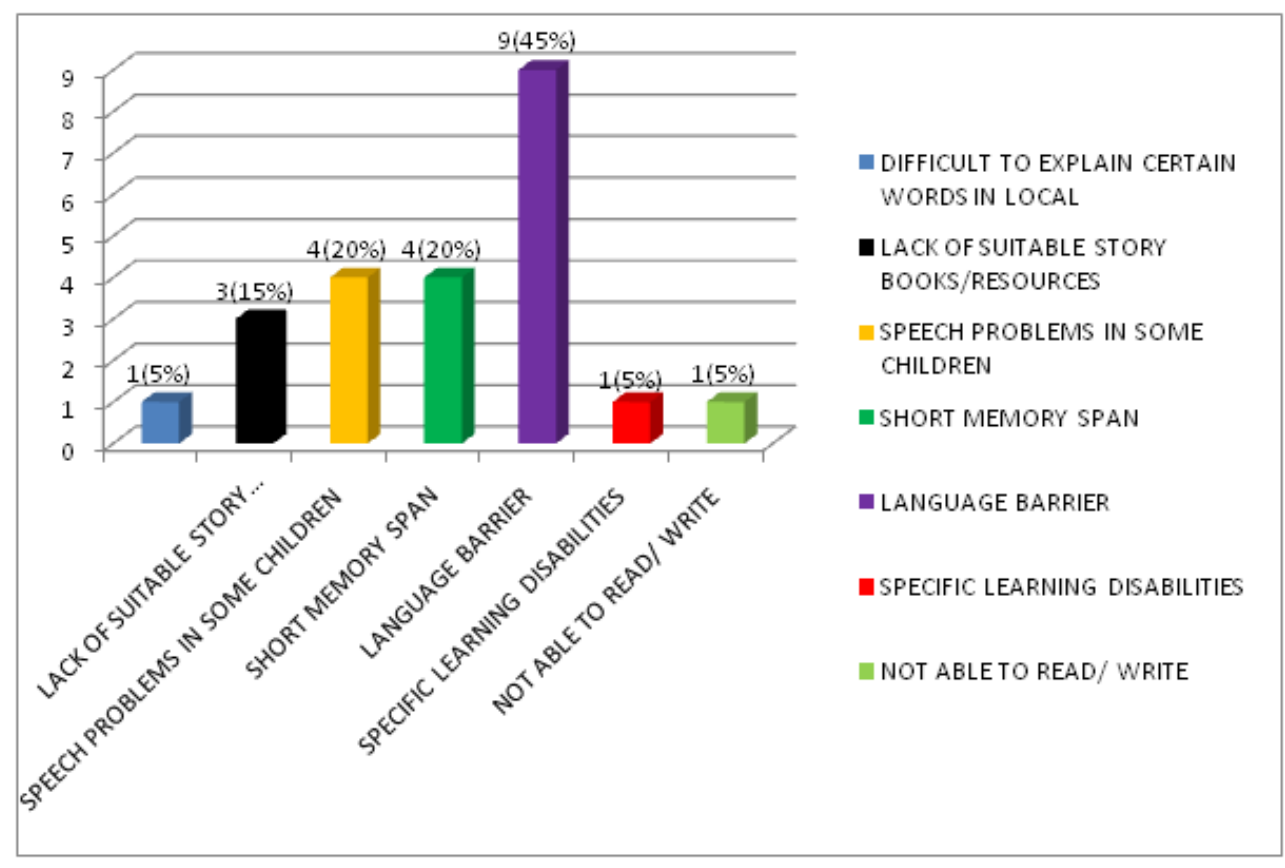

Figure10: Challenges experienced by teachers due to use of familiar language to teach

Figure 9 presents some of the challenges teachers have noticed during the process of teaching using familiar language. One respondent stated that explaining certain concepts which are in English to familiar language is a challenge, 3 respondents pointed out that lack of suitable teaching and learning materials for this category of learners is a challenge to teach them in a familiar language, 4 said speech problems in some learners with intellectual disabilities is also a challenge to teach them reading, another 4 said the short memory span of children with intellectual disabilities is yet another problem to the teaching of reading in a familiar language. Language barrier scored the highest, 9 respondents alluded to the fact that language is a major barrier to teach reading to learners with intellectual disabilities as most of the learners do not use the language used to teach when at home, hence causing 
Use of Familiar Language to Enhance Reading Skills in Learners with Mild Intellectual Disabilities in Early Grades Primary Schools in Southern Province of Zambia

challenges for the child to learn. Two respondents stated that some children have specific learning disabilities in addition to the intellectual disability and this myriad of disabilities makes it more difficult for them to learn in a different language. Three respondents stated more than just one challenge, which takes the number to be more than the actual number of respondents.

Teaching strategies to enhance reading among learners with mild intellectual disability

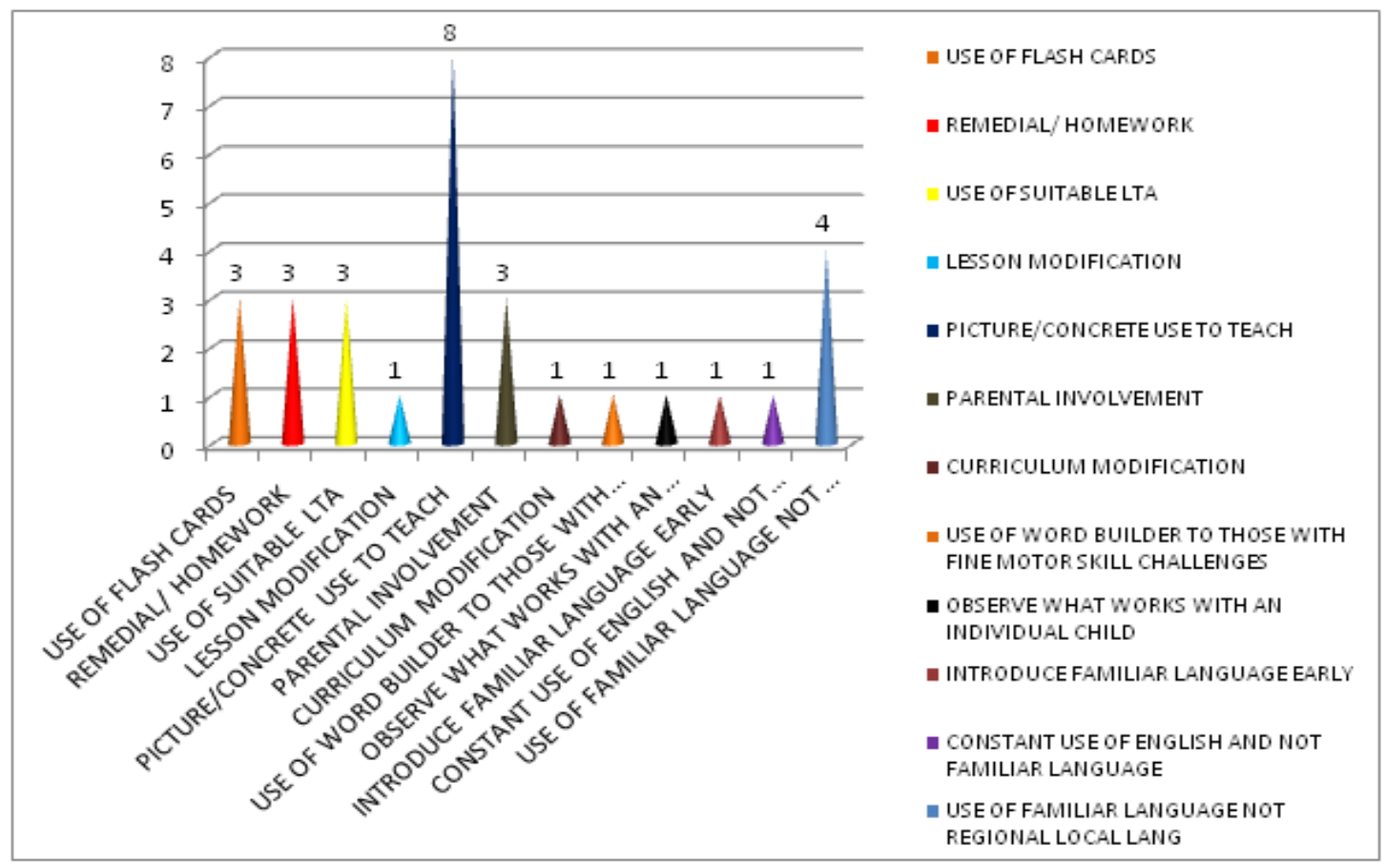

Figure11: presents the teaching strategies that most teachers felt could be more effective to teach learners with intellectual disabilities

\subsection{Interview Schedule with Head Teachers}

The head teachers from the sampled schools where subjected to an interview schedule just to solicit for detailed information and actually to validate the data collected through the questionnaires and the BASAT. The following where the responses from the head teacher of Maamba Mine Special School in Sinazongwe district;

The school has a total enrolment of 40 pupils who are intellectually disabled. 22 are boys and 18 are girls. Tonga is mostly used to teach in class and we have seen it to be effective because most children are able to read simple words and they show familiarity to most of the words however the challenge is on application. Mostly content presented in pictorial form is easy to read for most children with intellectual disability. We have also observed that from the time we started using Tonga to teach, most parents have shown more commitment in the learning of their children. Some parents have reported that they have observed some changes in their child as they are able to interact more with other children and also able to read some simple words, the thing which never use to happen. Apart from reading, the children are also able to do some daily activities like being sent to the market and transact with less challenges. However he also emphasized that there is need to concentrate more on the letter sounds.

At St Mulumba in Choma district, the head stated that the school has the following statistical enrolment of children with intellectual disabilities. There are 47 pupils of which 24 are boys while 23 are girl. Chitonga is used to teach in class and parental involvement is good. "Previously parents could not open up to give information regarding their child but not, there is more interaction between teachers and parents of children with intellectual disabilities. Parents even make follow-up to some of the school programs and activities. This is as a result of using the familiar language where parents freely express themselves.

Very few children are able to read and write, most of them are still below average. The challenge could be the use of local language since some of the children learn the language here at school; it is 
Use of Familiar Language to Enhance Reading Skills in Learners with Mild Intellectual Disabilities in Early Grades Primary Schools in Southern Province of Zambia

not the language they use at home. However the use of familiar language has enhanced social interaction among learners in school. Some children who use the familiar language at home have really improved and their participation in class has improved a lot.

To some extent, we still have challenges with some parents who still want donor aid for the education and well being of their children. The teaching strategies that seem to work well are actually the use of concrete object as teaching aids as children familiarize themselves to such materials. Matching of words to pictures is also quiet effective. However the most noticeable challenge is with children with multiple disabilities such as those with speech problems, it affects them to read properly. The head also said that "when you tap into their interest, children become very active and participation in class is very high". She also alluded to the speech/ language development problem in most children with intellectual disabilities as the factor that affects reading among these children and hence the need to concentrate on language development skills.

At Nakatindi, the head teacher stated that "being a pilot school for inclusive education, most cases of children with intellectual disability are mild and very few. These are in cooperated in the main stream. However the use of familiar language to teach in the lower grades have helped most children to improve their reading ability and there is more feedback from parents each time you give work to children to be helped at home. The reading levels generally are still low though there is a likely hood that most of them will break through in literacy. Parental involvement is still very low as most parent show very little concern for their children. The response is very bad even when they are called to school by the teachers."

The language barrier is another source of concern in the implementation of the familiar language. The familiar language to most children is Silozi and Chitonga. So the two languages contradict each other when it comes to reading however familiarization of things talked about in class and participation in class is good despite the two languages at play.

"David Livingstone secondary special unit only have 26 learners with intellectual disability and most of them are dyslexic. Due to this condition, it makes it very difficult for them to learn to read and worse with the use of local language", This is what the head of department said, whom the head teacher advised me to talk to. Use of Tonga to teach as the regional language is a challenge as most of the children do not use Tonga as their home language or language of play, most of them use Chinyanja. Therefore, to avoid this language barrier, we use English when teaching.

Parental involvement is still very bad as most parents do not respond effectively even when it comes to help their children at home with home work.

To us, the most effective teaching strategy is to teach these children according to their pace groups and constantly use English to teach.

Secondly it is also important to observe the interests and strength of the child and then teach them basing on that. Generally, the use of familiar language has not enhanced reading among children with intellectual disabilities.

\subsection{Observation of the Learning Environment}

The learning environment in all the sampled schools is very conducive as most classes show evidence that children are provided with reading materials in terms of charts and word cards for them to interact with at any time during and after the official learning time. This kind of learning environment helps learners to maximize their efforts to learn to read on their own and with friends. The walls are designed in such a way that children interact with reading materials all the time. The word picture matching is mostly used in all the schools selected for the study. However, the general surrounding is just like any ordinary school with nothing for children to read once out of the classrooms. The teacher pupil ratio in most classes observed was almost 1-5 in levels 3-4 and in level 1and 2 the ratio was about 1-8, with the majority of them being male.

\subsubsection{Parents Interview Schedule}

Most parents interviewed stated that they always find time to help their children with home work. Out of the five parents exposed to the interview schedule, three stated that they use Chitonga at home as their language while two uses Chinyanja. They all expressed satisfaction on the use of familiar 
language as it has made it easy for them to communicate with their children .One parent said "I find it easy to help my child with school work because I explain in a language my child understands better. This helps him to get some concepts with less challenge". All parents said that their children's social behavior had improved as they were able to interact freely with friends, family members and visitors who visit them. However they still have challenges with reading but do better with content matched with pictures. There is an improvement unlike before. This is what she said "previously we could not send him to go and buy things at the market but now since he has improved in his communication skills, he is able to go to the market and buy the items sent, he is also able to explain certain things clearly by using the language we use at home unlike in English".

The other parent expressed happiness on the use of familiar language to teach their child stating that" tandiyiide mebo taata onoo kubelesia chisyobo chesu iinga chaubauba kugwasya mwana ooyu kupandulula" Chkuwa chilashupa chita bakaiya chikolo. Meaning, "am not educated therefore it is easy for me to explain to my grandchild the reading text with pictorial presentations in our language, English is difficult". it is easy for them to explain to the child and that the child is able to identify pictures without problems. However she said that the child has just started school and that it is difficult to comment of the reading improvement never the less she was very optimistic the child will soon be able to read. "Most of the time, we give him some books with pictures because that's what he likes most". Other parents expressed sadness over the non availability of reading materials in a language their child is familiar to. This makes it difficult to find reading materials suitable for their children. On average, most parents interviewed, try to help their children with school work. However the economic status of most homes visited attest to the fact that most children with intellectual disabilities, where coming from homes which are economically disadvantaged and that could be one factor that can significantly affect their reading improvements due to unavailability of reading materials for the children to interact with other than the work given by the teachers as home work.

\subsubsection{Summary}

This study has revealed that despite the use of familiar language to teach reading to learners with intellectual disabilities, the reading levels remain low. However there is slight improvement on letter knowledge and pictorial reading comprehension, the difference or rather the improvement is very insignificant between the two academic years under which this study took place. The responses from the head teachers and parents during the interview schedule indicate that the use of familiar language to teach at the lower grades is a very good strategy as it is helping children with intellectual disabilities to develop socially and also to generate meaning to what the teachers talk about in class since the language is very familiar. Furthermore, parents also find it very easy to help their children with school work while at home. Some challenges teachers encounters have been discussed.

\subsubsection{Reading Levels of Learners with Intellectual Disability in Familiar Language}

The reading sub tests from the BASAT were used to test the reading skills of the learners. The reading sub tests were customized into chitonga, which is the most familiar language in the region. Each child took about 30-45 minutes to complete the test. This implies that adequate time was given to every learner to read without interference.

The findings clearly reveal that learners with mild intellectual disabilities still face significant challenges in reading despite being taught in a familiar language and this is actually in line with the findings of Neuman, (1999), who agrees to say "most children with intellectual disabilities lack the skill and knowledge of letter sound knowledge and alphabetical awareness". The two factors play a very significant role in the acquisition of early emergent literacy skills in children. Therefore it is against the premise that the researcher recommends that instead of the letter sound knowledge, teachers should concentrate so much on sound letter object identification (SLOI). This will help learners to acquire the concept of identifying objects in relation to sounds and letters as opposed to letter sound knowledge. The score in letter knowledge was very good (32\%) but very poor in letter sound knowledge which was $6 \%$. This means that there is little learning when children just memorize the letters of the alphabet without relating the sound to letters.

Letter sound knowledge was tested as follows; in the first place, the examiner had to say the sound in relation to the letter for the child and then later the examiner says the sound and the child had to point 
to the corresponding letter and then vice-versa. The total score in this category was extremely bad such that only five scores were made out of the possible maximum of 50. However the scores in this category are not surprising because if learners in level three (3) do not have adequate knowledge on the foundation skills for reading such as alphabetic knowledge letter sound knowledge and sound letter knowledge, how can a learner learn to read?. This performance is a true reflection that borders around the reading difficulties of learners with mild intellectual disabilities. The picture is similar to the data collected in the second phase under the letter sound knowledge. Most learners performed far much below the expectation though most researchers such as Tambulukani, et al, (2001), Sampa, (2003). Heugh, (2016) and Kashoki, (1990) assume that it is easy to relate the phonology of the word to its written form when the word is part of the Childs vocabulary (Tabors, et al, 2001\& Whitehurst, 2001). The case is different with learners with mild intellectual disabilities. Therefore, this is the area where teachers need to work hard if the intellectually disabled learners can learn how to read, using familiar language.

Under phonological knowledge, four skills were tested and these are syllable segmentation, initial sound identification, end sound identification and sound blending. There is a close correlation in the performance of learners with intellectual disabilities in the tests in the four skills as eluded to above. All the scores learners got were far below the average. For syllable segmentation the maximum score was $4(5 \%)$ in the first phase and the mean score in the second phase was 1.2361 with a standard deviation of 2.08612 better than the other scores probably it is because one syllable words and two syllable words are quite easy to repeat after someone as compared to words with more than two syllables. Further still, it is important to mention here that word cards in Chitonga were also used to reduce on the memory work load for the learners. Particularly this worked well with the blending task .When a word has been read to the child, it was then placed in front of him or her so that he/she can form a word. This brings out the fact that breaking some tasks into smaller units when teaching pupils with mild intellectual disability helps them to learn better. Carmine et al (2004) suggest that "in addition to reality based teaching and direct teaching, content for learners with intellectual disabilities should be broken down into very simple steps to ensure high success". Because of memory deficits, simple steps help them to grasp the concepts easily. Some scholars have also attributed reading deficits to problems with phonological processing in children with intellectual disabilities, (Conners, Atwell, Rosenquist, and Sligh, (2001). Gombert (2002) adds that deficits in phonological processing, contribute to the reading problems. Teachers have a task to work on the phonological aspect of the pupils if they are to impart reading skills to them. Despite the reading difficulties, children with mild intellectual disabilities have the ability to learn how to read though at a slow pace as compared to the typically developed peers.

A reading test from the BASAT was applied. Children were asked to read a series of words and two sentences. The categories fell into four groups: two letter words; one syllable words; two syllable words; and three syllable words. The scores were coded on a three point scale: 0 complete fail, 1 attempted to read and 2 able to read accurately. The maximum score was 24 points out of 38 . The performance of pupils in this category was below expectation considering the level of the tasks presented to them. It really shows how low the reading levels are for learners with mild intellectual disabilities as compared to "normal "pupils (MoE, 2008).In the second phase of data collection, the same reading sub test was applied to the learners, in Chitonga the familiar language. However the points were coded on the three points scale namely 0 stands for complete fail, 1 for attempted to read and 2 able to read correctly. The maximum core was 9 points out of 38 .

Reading comprehension was the only category where pupils showed some strength. Most pupils scored high under reading comprehension. The average score was actually 2 out of the maximum score of 4 in the first phase and in the second phase, the average score was 4 out of the maximum score of 4 . To the researcher this outcome indicates that pupils with mild intellectual disability can learn reading better if the basic reading materials for them are presented with pictures. This form of presenting reading tasks makes more sense to them than just words without pictures. They relate well with words to pictures and it assists them to read and at the same time get the meaning of what they read because they are able to see, this is evidence enough that the concept of sound letter object identification (SLOI) could be the most effective teaching strategy mashed together with teaching in a familiar language. To some extent this whole concept is in line with the hybridization concept by 
Banda (2008). Westwood, (2007) also suggests that the curriculum for learners with intellectual disabilities should be reality based. This is to ensure that there is meaning to what pupils see and read. Curriculum Frame Work, (2013) also adds to the fact that the use of familiar language to learners with intellectual disabilities will enhance reading and eventually academic success of learners. The findings are still consistent with the Zambia's National Assessment Survey of 2006 and also 2016 report on the low reading levels of most school going children in the lower grades and children with intellectual disabilities are not an exception. Table three shows the comparative correlation statistics between the previous reading levels and the current reading levels in a familiar language. The findings reveal that there is no significant difference between the previous and the current. This entails that there is still more to be done by both the teachers of learners with mild intellectual disabilities and the learners. However in some sub tests in Chitonga, learners showed some strength and if well explored, there can be improvement in the reading abilities. Otherwise, the descriptive statistics suggests that there was no significant difference in the reading abilities during the data collection periods.

\subsection{Impact of Using Familiar Language to Teach Learners with Mild Intellectual Disabilities}

In order to establish the impact or significance of the use of familiar language to teach learners with intellectual disabilities, teachers were exposed to a questionnaire and the following were the responses; figure 5 presented the information on whether teachers felt the use of familiar language had an impact on the enhancement of reading to learners with intellectual disabilities or not.60\% stated that it was average, meaning that the difference was very minimal as compared before using the familiar language. $20 \%$ said that teaching in a familiar language was very effective in the sense that most children were able to participate in the learning process during classroom contact periods unlike in the past. This is also alluded to by other researchers such as Afacan, et al (2017), Gombart, (2002) Tambulukan, (2011), Mkandawire,(2017), Dennis, (2008), and Kashoki, (2019), who also stated that children learn well in the language they are familiar to. The other $20 \%$ said that they were not sure whether using familiar language to teach learners with intellectual disabilities was effective or not effective.

The researcher also wanted to find out the teachers' competence levels in the language they were using to teach learners. This was done because teacher's competences in the language used to teach, has a direct co-relation to his or her classroom input while teaching. The responses were coded on a 4 point scale as follows; not at all $=1$, not sure $=2$, average $=3$ and very much $=4$ points. 7 respondents stated that they were very conversant with the language used to teach culminating into 28 points. This means that they had less challenges to teach children with mild intellectual disabilities in a familiar language and the likelihood that they were very innovative and resourceful in ensuring that they provided the necessary resources to the learners is very high, which eventually trickles down to high achievements. 11 respondents said they were average, translating into a total of 33 points and only 1 said was not competent with the familiar language used to teach the lower grades at the school coming to 2 points, None said was not sure respectively. The level of mastery of the language the teacher uses to teach has a bearing effect to the learners learning. This means that children cannot learn effectively because the teacher too requires learning the language. However most of the respondents were very competent with the familiar language meaning that they render the best of their service to the learners in terms of teaching reading. I would like to challenge teacher training colleges to ensure that the will be teachers are equipped with communicative skills during their training so that they are able to effectively and competently teach learners in the lower grades in a familiar language where ever they are deployed within Zambia for the benefit of young children and more to learners with intellectual disabilities.

\subsubsection{Reading Skills Enhanced as a Result of Teaching in a Familiar Language}

Through their observation and interaction with learners, teachers confirmed that despite the low reading abilities in the familiar language, children exhibited some reading skills that they have acquired as a result of using familiar language to teach them reading. $40 \%$ of teachers who took part in the study stated that most learners are able to read simple words. This success can be enhanced by creating a rich reading environment where learners can interact with reading materials even outside classroom time. This is in line with what Mwanza, (2012), Morrow, (1990); and Newman, (1993), reported on the need for a rich literacy environment. 5\% said that the use of familiar language has enhanced social interaction among children with intellectual disabilities. This is supported by what 
one parent said when she stated that "my child is now able to interact with friends and family members and visitors more that previously". This is evident enough that use of familiar language to teach also can enhance language development in the learners in the lower grades other than just reading proficiency. $25 \%$ are able to read words matched with pictures. Matching pictures with words does not only help learners to read but also develops analytical and comprehension skills in learners, which is very necessary in the academic advancement of learners. $20 \%$ postulated that most learners with intellectual disabilities have improved the skills of listening and speaking because they understand what the teachers says and are able to participate in class discussions and $10 \%$ said none of the skills have been enhanced as a result of using familiar language to teach the learners with intellectual disabilities. Catherine et al, (2005), states that, it is easy to apply phonological skills to reading in any language. Meaning that when children become aware of sound letter knowledge, then they can apply that skill in their reading, (Allor et al, 2014).

\subsubsection{Parental Involvement in Learning of their Children with Intellectual Disabilities as a Result of Teaching in a Familiar Language at School}

Figure 8, reveals the extent to which parents to children with disabilities get involved in the learning of their children with intellectual disabilities from the sampled schools. The majority of respondents stated that most parents are not often involved in the learning of their children, this constituted about $70 \%$ while $15 \%$ said parents were very often involved in the learning of their children from the time familiar language was implemented in school.10\% said parents were always involved in the learning of their children and 5\% stated that parents were not involved at all. It is very clear that the study revealed that most parents were involved in the learning of their children. This positive response could be as a result of the use of familiar language to teach them at school.

While student success largely relies on factors like study habits, school attendance, test-taking abilities and more, research is showing that parental involvement also has a large impact on student achievement in the classroom. In fact, according to the Academic Development Institute, parents who get involved with their children's education tend to demonstrate good parenting skills, volunteer their time in the school, communicate with faculty and take an active role in school-related decisionmaking. The Harvard Family Research Project of (2015) concluded that parental involvement is associated with higher student achievement. They found that student success was higher in a variety of areas including standardized test scores, grades and teacher ratings.. The types of parental involvement that had the greatest impact on student success were reading and communicating with one's child, as well as holding parental expectations. Children with intellectual disabilities stand to benefit more in the learning process when there is full parental involvement in their learning. The use of familiar language makes the acquisition of concept explicit and easy to learn. Catherine, et al, (2005), adds that evidence suggests that an encouraging home- literacy environment has a positive relationship with early literacy development. Researchers have advocated joint parent preschooler book reading, believing it to be beneficial for preparing children for formal reading instructions. This implies that the stories parents and peers tell to young children create a rich foundation for reading and this can be effective if parents take an active role in preparing their children with intellectual disabilities for future reading. Therefore it is important to encourage parents to take keen interest in the learning of their children with intellectual disabilities so as to enhance the acquisition of early literacy skills in children. Parents and siblings, including peers can create a rich literacy home environment that can encourage the development of emergent literacy skills in children with intellectual disabilities. The study revealed that parents are not always involved and this has an impact on the learning of reading to learners with intellectual disabilities.

\subsubsection{Means by Which Parents are Involved in the Learning of their Children with Intellectual Disabilities}

The study revealed that most parents were not always involved in the learning of their children; however it does not mean that they are not involved at all. Figure 8 presents the responses from the teachers on how they get parents involved in the learning of their children. 4 respondents (16) points said that home visits were one of the strategies used to involve parents to take part in the learning of their children with intellectual disabilities. 7 teachers stated that parents get involved in the learning of their children through home work assignment given to pupils by teachers, this amounted to 35 points, 
another 7 teachers stated that parents get engaged in the learning of their children through attending the parents teachers association meetings (PTA), this amounted to 14 points. 2 stated that parents were involved through open academic days and this response amounted to 6 points while none stated that parents were not involved in any way giving 0 points.

From the findings, it is clear that schools and teachers in particular have not done much in trying to get parents involved in the learning of their children with intellectual disabilities. Therefore it in important to come up with strategies that can get parents involved in the education of their children. According to Maryln, (2004), collaboration refers to the style or approach in the interaction between schools, parents, and community based on accomplishment of shared goals. Therefore, it is important that parents play an active role in the education of their children with intellectual disabilities. This collaboration has positive implications on learner academic achievements. With appropriate supports in place through collaborations with other stake holders such as parents in the learning of their children with intellectual disabilities, children can achieve high life in many different aspects. This collaboration does not only mean that parents need to go to school and see how their children learn, but what activities do they engage their children into while at home as a way to supplement the teachers efforts in teaching the child to read. It is very clear from the finding that very little has been done in this area of parent /teacher collaboration for the common good of the learners with intellectual disabilities. Schools should design a program, which will compel parents to engage into academic reading activities that are deliberately aimed at enhancing reading for their children whilst at home and to be evaluated by the school periodically.

\subsubsection{Challenges Teachers have Experienced While Teaching in a Familiar Language}

Figure 9 presents some of the challenges teachers have noticed during the process of teaching using familiar language. One $(5 \%)$ respondent stated that explaining certain concepts which are in English to familiar language is a challenge. This experience by the teacher entails that there are also gaps in the training of teachers of languages. It is possible that the teacher education curriculum for languages does not adequately address the issue of using familiar language to teach children how to read. Therefore, some teachers might be incompetent to teach effectively. This empirical evidence should help the teacher trainers to ensure that the much needed competencies by trainee teachers must be inculcated to them before they graduate so that they are able to teach with confidence. If teachers are not very conversant with the language when teaching reading, it subsequently affects the learners as well; therefore, teacher's competence in the language use is cardinal to the learning achievements of the learners. $15 \%$ of the respondents pointed out that lack of suitable teaching and learning materials for this category of learners is a challenge to teach them in a familiar language, Kioko, (2004), stated that teaching children with intellectual disabilities requires the adaptation of the entire general school curriculum so that the work presented to children with disabilities is suitable according to their learning. Therefore the inadequacy of suitable teaching and learning materials for children with intellectual disabilities in schools, defeats the efforts of trying to improve their reading skills through the use of familiar language. The assumption that children learn well when actual objects or semi abstract situations like pictures are made available to them cannot be achieved if there are no suitable learning materials in schools. Schools, government and other stakeholders should work together to ensure that the suitable teaching and learning materials for this category of learner are made available to schools so that the implementation of familiar language is further strengthened. $20 \%$ said speech problems in some learners with intellectual disabilities is also a challenge to teach them reading. Most children with intellectual disabilities exhibit a myriad of language and speech problems however, regardless of that, they are still able to communicate with others including teachers. However the speech problem cannot be over looked as speech and language development or language proficient is an attribute to reading proficiency in children. It is therefore important that language / speech development skills go side by side with the reading activities in order to attain reading proficiency in children with intellectual disabilities. Another $20 \%$ of respondents said that short memory span of children with intellectual disabilities is yet another problem to the teaching of reading in a familiar language. Language barrier scored the highest, $45 \%$ respondents alluded to the fact that language is a major barrier to teach reading to learners with intellectual disabilities as most of the learners do not use the language used to teach when at home, hence causing challenges for the child to learn. This language barrier was also observed by Mwanza, (2012). It is common in most cosmopolitan settings 
such as towns. The language that children are familiar to, is not the familiar language used for teaching at school. However there is a strong link of similarities in Bantu speaking languages of which Zambian languages are part of the Bantu speaking people. Even if there are some differences but most pronunciations of lexical items and consonant and vowels are almost the same, Sepell, (2014) and that it should be easy for children to learn and teachers to teach effectively. On the other hand, the approach should be very flexible to accommodate other languages in the teaching so that children can understand what the teacher is explaining. For instance in chinyanja a dog is called Galu, while the urban Nyanja a Dog is called Imbwa. In chitonga, a dog is called Mubwa while the Tonga of the Zambezi valley calls a dog as Munkala. However, using the (SLOI), an audio and video approach to teaching reading, children will familiarize the words by identifying the object. This will still help learners to understand the concept regardless of the home language one speaks, otherwise the assumption remains that the familiar language concept still works when effectively implemented. 5\% of the respondents stated that some children have specific learning disabilities in addition to the intellectual disability and this myriad of disabilities makes it more difficult for them to learn in a different language. However the presence of other disabilities children with intellectual disability might have can only be a challenge to learning but it does not mean that they cannot learn in another language. The fact that the assumption of this study is based on familiar language as a tool to use on order to enhance reading levels among children with intellectual disabilities. Let the children be taught how to read in a language familiar to them regardless of the presence of other learning disabilities such as dyscalculia and dysgraphia. Three (15\%) of the respondents stated more than just one challenge, which takes the number to be more than the actual number of respondents.

\subsubsection{Teaching Strategies to Enhance Reading among Learners with Mild Intellectual Disabilities}

Figure 11 clearly shows that children with intellectual disabilities learn well and faster when content is presented in a familiar form meaning in a pictorial form of concrete objects. $40 \%$ of respondents (teachers) stated that reality based teaching to children with intellectual disabilities does not only enhances reading, but also comprehension. This revelation strengthens further the assumption that sound letter object identification (SLOI) could be the most effective teaching strategy to teach emergent literacy skills in children at early grade level. Phonology and letter knowledge are very important pre-requisites to reading proficiency and that the strategy of sound letter object identification can greatly help children to learn how to read. What they see in the community and at home is then brought in class and then written in worlds that sound exactly in the language he/she understands better. This can really speed up the rate at which learners learn how to read in a familiar language because there is a lot of meaning attached to what they hear and see in and outside class time when words are matched with concrete objects of pictures. Kioko 2004, adds that the use of real materials or actual tools in natural environments is an essential component in the effective instruction to students with intellectual disabilities. An illustration is given that a high school student would use a calculator to work mathematics problem where as an elementary student may be more likely to use counting blocks. $15 \%$ of the respondents stated that the use of flash cards is also one teaching strategy that can enhance reading to learners with intellectual disabilities, another 15\% stated that intensive use of remedial work and also use of suitable teaching and learning materials can enhance reading proficiency among learners with intellectual disabilities. One respondent expressed confidence that constant use of English is the only way to enhance reading skills among learners with intellectual disabilities. $20 \%$ of the respondents acknowledged the use of familiar language to be effective and not the regional or familiar language. This was confusing to the researcher in that its implication was that each child be taught in a language he/she is familiar with. However the concept and its assumption is that the language most learners use is more zonal oriented and that it is from this fact that such a language is to be used for teaching since it is understood by most of the children. In any case there is still need to further explore the possibility of teaching every child in the language familiar to that child in the near research by researchers. Others said that lessons should be modified by applying the concept of task analysis. This is where larger chunks of content are broken into simpler units so that children can learn one thing at a time. Gombat, (2002); Romski and Morris, (2010) further states that a mult-component intervention can improve student's phonemic awareness skills, which can help learners to develop their emergent literacy skills. Recent research is demonstrating that with an integrated and systematic approach to teaching reading to learners with mild intellectual disabilities, 
Use of Familiar Language to Enhance Reading Skills in Learners with Mild Intellectual Disabilities in Early Grades Primary Schools in Southern Province of Zambia

learners can successfully combine and separate skills of phonemic awareness and letter sound correspondence to decode unfamiliar words, (Courtade, Gibbi and Flower, 2008).

\subsubsection{Interview Schedule with Head Teachers}

The head teachers from the sampled schools where subjected to an interview schedule just to solicit for detailed information and actually to validate the data collected through the questionnaires and the BASAT. The following where the responses from the head teacher of Maamba Mine Special School in Sinazongwe district;

The school has a total enrolment of 40 pupils who are intellectually disabled. 22 are boys and 18 are girls. Tonga is mostly used to teach in class and we have seen it to be effective because most children are able to read simple words and they show familiarity to most of the words however the challenge is on application. Mostly content presented in pictorial form is easy to read for most children with intellectual disability. We have also observed that from the time we started using Tonga to teach, most parents have shown more commitment in the learning of their children. Some parents have reported that they have observed some changes in their child as they are able to interact more with other children and also able to read some simple words, the thing which never use to happen. Apart from reading, the children are also able to do some daily activities like being sent to the market and transact with less challenges. However he also emphasized that there is need to concentrate more on the letter sounds. It is evident enough from the responses that children are making progress more on the social domain unlike cognitive however like the head said, there is need to enhance the sound letter knowledge and object identification in order to enable children with mild intellectual disabilities make progress in their reading skills.

At St Mulumba in Choma district, the head teacher stated that the school had the following statistical enrolment of children with mild intellectual disabilities. There are 47 pupils of which 24 are boys while 23 are girl. Chitonga is used to teach in class and parental involvement is good. "Previously parents could not open up to give information regarding their children but now, there is more interaction between teachers and parents of children with intellectual disabilities. Parents even make follow-up to some of the school programs and activities. This is as a result of using the familiar language where parents freely express themselves.

Very few children are able to read and write, most of them are still below average. The challenge could be the use of local language since some of the children learn the language here at school; it is not the language they use at home. However the use of familiar language has enhanced social interaction among learners in school. Some children who use the familiar language at home have really improved and their participation in class has improved a lot. Because of the enhanced social interactions, some children with intellectual disabilities have even learnt how to communicate using sign language with those children with hearing disability.

To some extent, we still have challenges with some parents who still want donor aid for the education and well being of their children. The teaching strategies that seem to work well are actually the use of concrete object as teaching aids as children familiarize themselves to such materials. Matching of words to pictures is also quiet effective. However the most noticeable challenge is with children with multiple disabilities such as those with speech problems, it affects them to read properly. The head also said that "when you tap into their interest, children become very active and participation in class is very high". She also alluded to the speech/ language development problem in most children with intellectual disabilities as the factor that affects reading among these children and hence there is need to concentrate on language development skills.

At Nakatindi, the head teacher stated that "being a pilot school for inclusive education, most cases of children with intellectual disability are mild and very few. These are in cooperated in the main stream. However the use of familiar language to teach in the lower grades have helped most children to improve their reading ability and there is more feedback from parents each time you give work to children to be helped at home. The reading levels generally are still low though there is a likely hood that most of them will break through in literacy." Parental involvement is still very low as most parent show very little concern for their children. The response is very bad even when they are called to school by the teachers. 
Use of Familiar Language to Enhance Reading Skills in Learners with Mild Intellectual Disabilities in Early Grades Primary Schools in Southern Province of Zambia

The language barrier is another source of concern in the implementation of the familiar language. The familiar language to most children is Silozi and Chitonga. So the two languages contradict each other when it comes to reading however familiarization of things talked about in class and participation in class is good despite the two languages at play.

"David Livingstone secondary special unit only have 26 learners with intellectual disability and most of them are dyslexic. Due to this condition, it makes it very difficult for them to learn to read and worse with the use of local language", This is what the head of department said, whom the head teacher advised me to talk to. Use of Tonga to teach as the regional language is a challenge as most of the children do not use Tonga as their home language or language of play, most of them use Chinyanja. Therefore, to avoid this language barrier, we use English when teaching.

Parental involvement is still very bad as most parents do not respond effectively even when it comes to help their children at home with home work.

To us, the most effective teaching strategy is to teach these children according to their pace groups and constantly use English to teach.

Secondly it is also important to observe the interests and strength of the child and then teach them basing on that. Generally, the use of familiar language has not enhanced reading among children with intellectual disabilities at our unit. There is controversy of familiar language to use at this school. The school is just in the heart of Livingstone town and the issue of language becomes a problem more to the challenge of learners with intellectual disabilities. The languages used at homes are different for most children and when subjected to read in Chitonga for a reading test, the performance was extremely poor. The researcher adopted Chitonga being in the Southern region of Zambia, where Chitonga is so dominant and by policy it is the language children learn as a subject other than English in all the schools. However this phenomena helps to understand that what is familiar language to others might not be familiar to some despite being in the same locality but what is common should be adopted for the good of children learning.

\subsection{Observation of the Learning Environment}

The learning environment in all the sampled schools is very conducive as most classes show evidence that children are provided with reading materials in terms of charts and word cards for them to interact with at any time during and after the official learning time. This kind of learning environment helps learners to maximize their efforts to learn to read on their own and with friends. The walls are designed in such a way that children interact with reading materials all the time. The word picture matching is mostly used in all the schools selected for the study. However, the general surrounding is just like any ordinary school with nothing for children to read once out of the classrooms. This should not be the case; children with intellectual disabilities need a rich literacy stimulating environment so that the development of emergent literacy skill is stimulated by the environment. The teacher pupil ratio in most classes observed was almost 1-5 in levels 3-4 and in level 1and 2 the ratio was about 1-8, with the majority of them being male. The language used to teach was Chitonga. The researcher observed that children participated during the learning time although some children did not use the Chitonga to communicate with the teacher but some could be head talking in Nyanja. At David Livingstone secondary, the teachers taught their children in English. When asked, the researcher was told that it was the language most children know better and even use at home. Socially the learners were able to talk in English but reading is still a problem. The researcher also took interest to look at most of the teaching and learning materials that children with intellectual disabilities used, there is a serious shortage of learning materials and that the only available books are not very suitable to learners with intellectual disabilities. The books where just adopted from the books "normal" children use without taking into consideration the typical development nature of children with intellectual disabilities. To this effect, the materials are not helping this category of learners to learn to read.

\subsubsection{Parents Interview Schedule}

Most parents interviewed stated that they always find time to help their children with home work and also with other academic work like teaching them how to do certain thing like activities for daily living (ADL), each time they are at home. Out of the five parents exposed to the interview schedule, three stated that they use Chitonga at home as their language while two uses Chinyanja, this parent 
was interviewed from Choma. All the parents interviewed were literate except for one who happened to be the grandmother to the child who was illiterate. They all expressed satisfaction on the use of familiar language as it has made it easy for them to communicate with their children .One parent said "I find it easy to help my child with school work because I explain in a language my child understands better. This helps him to get some concepts with less challenge". Even his siblings are able to help him in certain things like explaining some mathematical concepts. All parents interviewed said that their children's social behavior had improved as they were able to interact freely with friends, family members and visitors who visit them. However they still have challenges with reading but do better with content matched with pictures. There is an improvement unlike before. This is what she said "previously we could not send him to go and buy things at the market but now since he has improved in his communication skills, he is able to go to the market and buy the items sent, he is also able to explain certain things clearly by using the language we use at home unlike in English".

The other parent expressed happiness on the use of familiar language to teach their child stating that" tandiyiide mebo taata onoo kubelesia chisyobo chesu iinga chaubauba kugwasya mwana ooyu kupandulula" Chkuwa chilashupa chita bakaiya chikolo. meaning, "am not educated therefore it is easy for me to explain to my grandchild the reading text with pictorial presentations in our language, English is difficult because am not educated". It is easy for them to explain to the child and that the child is able to identify pictures without problems. However she said that the child has just started school and that it is difficult to comment of the reading improvement never the less she was very optimistic that the child will soon be able to read. "Most of the time, we give him some books with pictures because that's what he likes most". Other parents expressed sadness over the non availability of reading materials in a language their child is familiar to. This makes it difficult to find reading materials suitable for their children. On average, most parents interviewed, try to help their children with school work. However the economic status of most homes visited attest to the fact that most children with intellectual disabilities, where coming from homes which are economically disadvantaged and that could be one factor that can significantly affect their reading improvements due to unavailability of reading materials for the children to interact with other than the work given by the teachers as home work.

\subsubsection{Summary}

This chapter has revealed that despite the use of familiar language to teach reading to learners with intellectual disabilities, the reading levels remain low. However there is slight improvement on letter knowledge and pictorial reading comprehension. The responses from the head teachers and parents during the interview schedule indicate that the use of familiar language to teach at the lower grades is a very good strategy as it is helping children with intellectual disabilities to develop socially and also to generate meaning to what the teachers talk about in class since the language is very familiar. This is in line with what most teachers suggested as a teaching strategy. Furthermore, parents also find it very easy to help their children with school work while at home. Some challenges that teacher's encounter as they teach in the familiar language have also been highlighted such as inadequate materials suitable for learners with intellectual disabilities, language barrier to some learners and teachers as well. Some mitigation measures to some challenges were also suggested in this study.

\section{CONCLUSION AND RECOMMENDATIONS}

This research has uncovered evidence based on the benefits of familiar language to learners with mild intellectual disabilities that has hitherto gone unreported for some time since the implementation of the language policy in schools in Zambia. Research findings on the use of familiar language to children without mild intellectual disabilities have shown that there is great improvement in reading when children are taught in a familiar language they know. However, despite the use of familiar language to teach reading to learners with intellectual disabilities, the reading levels remain very low. However there indications of improvements on letter knowledge and reading comprehension, where content is presented in pictorial form. The core under this category was very high during the BASAT sub test on the learners. Social skills for interaction have been enhanced due to the use of familiar language. Most responses from parents and teachers revealed that children were more able to interact during learning and play time. Parental involvement in the learning of their children with intellectual disabilities has also improved. Most parents interviewed attested to the fact that the use of familiar 
language has made it easy for them to explain certain concepts to their children. However to further enhance the participation of parents in the learning of their children with intellectual disabilities, most teachers and schools in general should scale up their efforts and strategies of getting parents involved in the learning of their children. The findings further highlighted on a number of challenges that teachers encounter as they implement language policy as enshrined in the new revised curriculum. Among some of the challenges is the language barrier, some of the children do not use the familiar language used for teaching at school and as such it is still a challenge for them to improve their reading abilities. This challenge is not only with the learners, but also the teachers. The researcher took interest to find out on how conversant the teachers were with the language used to teach; in this case it is (Chitonga). Some teachers are not very competent with the language and that has an effect on the learning of the learners with intellectual disabilities. Most teachers need help to learn the language so that they can effectively execute their duties of teaching to learners with intellectual disabilities. Another challenge alluded to by most teachers was lucky of suitable and adequate teaching and learning resources for learners with intellectual disabilities. Suitable learning materials play a very important role in the learning of children in the early grades and worse children with intellectual disabilities. They need to interact with a lot of reading materials in a familiar language in order to familiarize them with what they learn in class to what they know from home. The presence of other disabilities in children with intellectual disabilities was also another concern raised by the teachers. Most of the children with intellectual disabilities have other disabilities such as language difficulties, Autistic, and other specific learning disabilities like Dyslexia, which make the learning very difficult. However, despite the above outlined challenges, teachers suggested some of the teaching strategies that they think could be most effective to teach reading to learners with intellectual disabilities. Some of the strategies are word picture matching which most teachers said the approach is very effective as most children are able to identify the object say it in the language they know. In the process they learn to read the names of such objects easily. Based on this principle, the researcher suggested that phonological knowledge and letter sound knowledge are important aspects in the learning of reading of any child. Therefore the letter sound knowledge advocated for by most practitioners is quiet mechanical and encourages children just to sing the alphabet and not really coming to the knowledge of phonemes and phonology in their reading lessons. Therefore employing the Sound Letter Object Identification (SLOI) approach could help enhance the development of emergent literacy skills in learners with intellectual disabilities. The reason being that children will familiarize with the sound to a letter of the alphabet, and then associate that sound to an object and at the same time be able to identify that object. Now, because the language is familiar, it makes it easy to identify most of the words associated to objects. Furthermore, this approach could be more effective to the teaching of reading in a familiar language because most of the senses in the child are activated or rather concentrate on one aspect hence maximizing the time spent on learning of one aspect. From this research, it is clear that the use of familiar language has not changed or enhanced the reading levels of This research has pointed out some of the measures and strategies that can be implored in the teaching of reading to intellectually disabled pupils in order to enhance the reading levels of the pupils and actually improve the teaching strategies of the teachers.

\section{RECOMMENDATIONS}

Based on the research findings, below are some of the recommendations made by the researcher:

- Intellectual disability is among the most prevalent disabilities in most schools. Therefore the Ministry of Education should equip trainee teachers in teacher training institutions with special skills to teach pupils who are intellectually disabled in a familiar language competently

- The reading levels among learners with intellectual disabilities are still insignificant however, there are indicators that if the use of familiar language can be implemented for a much longer period, as opposed to what is stated in the policy document that grade one to four (1-4). Quick transition into English could seriously affect their learning for they take long to conceptualize concepts.

- Parental involvement in the learning of their children should be strengthened further. The home environment can positively impact on the learning of a child with intellectual disabilities if parents work in collaboration with teachers. 
Use of Familiar Language to Enhance Reading Skills in Learners with Mild Intellectual Disabilities in Early Grades Primary Schools in Southern Province of Zambia

- Parents should ensure that there is early exposure to suitable reading materials to Pupils with intellectual disabilities through creating a stimulating reading home environment.

- The Ministry of Education and indeed other stake holders in the education of children with disabilities should consider making available suitable and quality teaching and learning resources for children with intellectual disabilities

\section{Suggestions for Further Research}

- In future, there should be research on the selection criteria of the familiar language to use more in urban schools in order to minimize on the effects of language barrier to learners and their teachers.

- In future, there should be research on the trainee teacher competences in teaching Zambian languages in Zambia

- There should be research on the schools preparedness to enroll learners with intellectual disabilities

\section{REFERENCES}

[1] Adams, M. J. (1990). Beginning to read: Thinking and learning about print. Cambridge, MA: MIT Press.

[2] Ader, H.J. (2008). Phases and initial steps in data analysis. Netherlands: Johannes Van Kessel Publishing.

[3] Bandura, A. (1977). Social Learning Theory. Englewood Cliffs, NJ: Prentice Hall.

[4] Catherine McBridge-chang \& Jasmine yuet-Han lau (2005). Home literacy and chines reading in Hong Kong children. Vol 16

[5] Center for Studies in Inclusive Education (2016). The UNESCO Salamanca Statement. Retrieved from http://www.csie.org.uk/inclusion/UNESCO-Salamanca-shtml

[6] Cheatham, J.P., and Champlain, T.M., (2010). Individualized research- based reading instruction for students with intellectual disabilities: Success stories. Teaching Exceptional Children; 42 (3), 6-12

[7] Chitiyo, M., \& Muwana, F.C. (2018). Positive Developments in Special Education in Zambia and Zimbabwe. International journal of whole schooling,14(1) 93-115

[8] Cruickshank, W.M., et al. (1961). A teaching method of brain injured and hyperactive children. Syracuse, NY: Syracuse University press.

[9] Cunningham, A.E., \& Stanovich, K.E. (1998). What reading does for the mind: America Education 22 (Spring/Summer), 8-15

[10] Dennis, B. (2008). Education for All (EFA) and the indigenous knowledge systems. University of Nottingham, E print.

[11] Dominik, T., \& Stephen, G. (2013). Research proposal and Dissertation writing. Dar es Salaam: Nyambari Nyangwine publishers.

[12] Education for All, (2007). Education for All. Global monitoring Report. Strong Foundations, Early childhood care and Education. Paris: UNESCO.

[13] Herbert, M. (2003). Typical and Atypical Development from conception to Adolescence: London, UK.BPS Blackwell Books.

[14] Kashoki, M.E., (2019). The African University: Towards the Linguistic Liberation of Africa. Lusaka: UNZA Press.

[15] Kaskoki,M.E., (1990). The factor of language in Zambia. Lusaka: Kenneth Kaunda foundation

[16] Katims, D. S. (2000). Literacy instruction for people with mental retardation: Historical highlights and contemporary Analysis. Education and Training in Mental Retardation and Developmental Disabilities, 35(1), 3-15.

[17] Katims, D. S. (2001). Literacy assessment of students with mental retardation: An exploratory investigation. Education and Training in Mental Retardation and Developmental Disabilities, 36(4), 363-372.

[18] Koch, A. (2008). The cultural technique of reading in schools for students with intellectual disabilities: learning to read without phonological awareness? Justus-Liebig Universität Gießen, Gießen. Retrieved from http://geb.uni-giessen.de/geb/ volltexte/2018/6247/pdf/KochArno-2008-28-05.

[19] Matafwali, B. (2005). Nature and Prevalence of Reading difficulties in the third Grade. Lusaka rural and urban schools. Lusaka: University of Zambia. Unpublished M.Ed dissertation.

[20] Matafwali, B. (2010). The Relationship Between Oral Language and Early Literacy Development: A Case of Zambian Languages and English, $\mathrm{PhD}$ Thesis, University of Zambia, Lusaka

[21] Micheal, s. Roseburg, David, L. Westling and James Mileskey (2011). Special Education for today`s teachers. New Jersey: Pearson Education inc 
Use of Familiar Language to Enhance Reading Skills in Learners with Mild Intellectual Disabilities in Early Grades Primary Schools in Southern Province of Zambia

[22] McGregor, S.L.T, \& Murname, J.A. (2010). Paradigm, Methodology and method: Intellectual integrity in consumer scholarship. International journal of consumer studies. 34(4): 419-427.

[23] Ministry of Education, (1992). Focus on Learning. Lusaka: Government printers

[24] Ministry of Education, (1996). Educating our future. Policy Reform document. Lusaka: Zambia Education Publishing House.

[25] Ministry of Education. (2006). National Assessment Report: Lusaka: Zambia Education Publishing House.

[26] Ministry of Education, (2008). National Literacy Survey report. Lusaka: Zambia Education Publishing House.

[27] Ministry of Education, (1996). Educating our future. Policy Reform document. Lusaka: Zambia Education Publishing House.

[28] Ministry of General Education (2017). Zambia National Assessment Survey report 2016: Learning Achievements at the junior secondary School level. Lusaka: MoGE.

[29] Muzumara,P.M (2011). Teacher Competencies for Improved Teaching and Learning. Lusaka: Bhuta Publishers.

[30] Mwanza, D., (2012). The language of initial literacy in a cosmopolitan Environment: A case of Chinyanja in Lusaka District. Unpublished MED Dissertation. Lusaka: University of Zambia

[31] Paula,Lyytinen, (1998). European journal of psychology of education. Vol; xii, 297-308

[32] Piaget, J. \& Inhelder, B. (1964). The Childs conception of space: London. Rout ledge and Kegan Paul.

[33] Pringle, M.K., \& Varma, V.P. (1974). Advances in Educational Psychology. UK: University of London Press

[34] Sepell, R. (2014). Promotion of Literacy in Sub-Saharan Africa: Goals and prospects of CAPOLSA at the University of Zambia. Human Technology, 10, 22-38.

[35] Sepell,R. \& Simatende, B. (2016). Contextual responsiveness: An enduring challenge for educational assessment in Africa. Journal of intelligence, 4 (3).

[36] Stanovich, K.E., \& Siegel, L. (1994). Phenotypic profile of children with intellectual disabilities: A regression based test of the phonological- score variable-difference model. Journal of Educational psychology, 86, 24-53.

[37] Stanovich, K.E., (1993). Does reading make you smarter? Literacy and the development of verbal intelligence. Advances in child development and Behavior, 24.133-180

[38] Tambulukani (2015). First language teaching of initial reading: Blessing or curse for Zambian children under primary reading program. PhD thesis University of Zambia Lusaka.

[39] Torgesen, J.K. (2000). Individual differences in responses to early interventions in reading: Learning Disabilities Research and practice, 15, 55-64.

[40] Turnbull, A. Turnbull, R., \& Wehmeyer, M.L., (2007). Exceptional lives-special education in today`s schools. Upper saddle River, NJ: Pearson Merrill Hall.

[41] White, C.J., (2005). Research; a practical Guide. Pretoria: Ithuthuko investment publishing.

[42] William, E. (1993). Report on Reading in English in primary schools in Zambia. ODA Research project 4770. Serial No, 5 University of Reading, April, 1993.

\section{AUTHOR BIOGRAPHY}

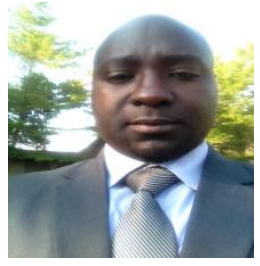

Kasebula Francis is a lecturer at David Livingstone of Education. He is a holder of a Masters degree in Special Education and now a PhD STUDENT at the University of Zambia

Citation: Kasebula Francis, et.al. "Use of Familiar Language to Enhance Reading Skills in Learners with Mild Intellectual Disabilities in Early Grades Primary Schools in Southern Province of Zambia". International Journal of Humanities Social Sciences and Education (IJHSSE), vol. 6, no.12, 2019, pp. 1-41. doi: http://dx. doi.org/10.20431/2349-0381.0612001.

Copyright: () 2019 Authors. This is an open-access article distributed under the terms of the Creative Commons Attribution License, which permits unrestricted use, distribution, and reproduction in any medium, provided the original author and source are credited. 